TRANSACTIONS OF THE

AMERICAN MATHEMATICAL SOCIETY

Volume 349, Number 6, June 1997, Pages 2391-2426

S $0002-9947(97) 01732-7$

\title{
THE STRETCH OF A FOLIATION AND GEOMETRIC SUPERRIGIDITY
}

\author{
RAUL QUIROGA-BARRANCO
}

\begin{abstract}
We consider compact smooth foliated manifolds with leaves isometrically covered by a fixed symmetric space of noncompact type. Such objects can be considered as compact models for the geometry of the symmetric space. Based on this we formulate and solve a geometric superrigidity problem for foliations that seeks the existence of suitable isometric totally geodesic immersions. To achieve this we consider the heat flow equation along the leaves of a foliation, a Bochner formula on foliations and a geometric invariant for foliations with leafwise Riemannian metrics called the stretch. We obtain as applications a metric rigidity theorem for foliations and a rigidity type result for Riemannian manifolds whose geometry is only partially symmetric.
\end{abstract}

\section{INTRODUCTION}

The symmetric spaces of noncompact type and their quotients have been a source of a great deal of study. One of the first important steps into the understanding of the relations between the geometry and topology of compact quotients of such spaces was given by Mostow's strong rigidity theorem.

Theorem (Mostow [18]). Let $X$ and $Y$ be compact quotients of symmetric spaces of noncompact type with the same fundamental group. If $X$ has no closed two dimensional local factor, then $X$ and $Y$ are isometric up to normalizing constants.

Later on, Mostow's theory was extended with Margulis' work by considering the fundamental group of a quotient of a symmetric space as a discrete subgroup of a semisimple Lie group, and then proceeding to study the extension problem for homomorphisms defined on such subgroups. A particular case of the main results of Margulis translated to the geometry of symmetric spaces is the following:

Theorem (Margulis). Let $\tilde{X}, \tilde{Y}$ be symmetric spaces of noncompact type. Assume that $\tilde{Y}$ is irreducible and $\operatorname{rank}(\tilde{X}) \geq 2$, and let $X=\tilde{X} / \Gamma$ be an irreducible finite volume quotient. If $\rho: \Gamma \rightarrow \operatorname{Isom}(\tilde{Y})$ is a representation with Zariski dense image, then there is a $\rho$-equivariant totally geodesic map $\tilde{X} \rightarrow \tilde{Y}$.

From this point, there have been several routes followed in an attempt to generalize or extend the superrigid properties of semisimple Lie groups without compact factors and the symmetric spaces associated to them. In the context of Lie group actions, Zimmer [24] extended Margulis' superrigidity to a cocycle superrigidity

Received by the editors December 15, 1994 and, in revised form, December 2, 1995.

1991 Mathematics Subject Classification. Primary 53C12, 58E20; Secondary 58G11, 28 A33.

Research supported by the Andrew Corporation, CONACYT-Mexico, COFAA-IPN-Mexico and SNI-Mexico. 
which has proved useful in the study of actions of semisimple Lie groups without compact factors.

On the other hand, from a geometric point of view there are several natural generalizations to both Margulis' superrigidity and Mostow's strong rigidity. In particular it was proved that the strong rigidity of symmetric spaces is a phenomenon that takes place in the broader context of manifolds with nonpositive curvature:

Theorem (Gromov [2], Ballmann-Eberlein [1]). Let $X$ be a finite volume irreducible quotient of a symmetric space of rank $\geq 2$. Let $Y$ be a complete Riemannian manifold of bounded nonpositive sectional curvature and finite volume. If $\pi_{1}(X) \cong$ $\pi_{1}(Y)$, then $X$ and $Y$ are isometric up to normalizing constants.

In all of the above results the upshot is that for compact (or even finite volume) quotients of suitably chosen symmetric spaces of noncompact type the geometry is determined by the topology. Such a general property is attained by proving the existence of isometric totally geodesic immersions.

It also has been found in general that compact manifolds with smooth foliations carrying a smooth leafwise Riemannian metric, i.e. a smooth Riemannian metric on the bundle of vectors tangent to the leaves, can be considered as a compact model similar to a compact Riemannian manifold. Even though a leaf in one such foliation is not in general a compact quotient of its universal cover, it has been possible to extend results given for compact manifolds to this kind of foliations with leafwise Riemannian metrics. One of the many examples of this remark is a strong rigidity result for foliations with leaves given by Riemannian quotients of symmetric spaces of non-compact type that was proved by Zimmer [22]. For a complete discussion of this result and the concepts involved we refer to [22] and [23]. Here we want to point out that, given a strong rigidity for foliations and the fact that the superrigidity of symmetric spaces has been proved to hold in broader contexts, it is natural to consider a corresponding superrigidity problem for foliations. Based on these remarks we formulate the following:

Problem A (Superrigidity for foliations). Let $M$ and $N$ be compact smooth manifolds with smooth leafwise Riemannian metrics and let $f: M \rightarrow N$ be a smooth leaf preserving map. Assume each leaf of $M$ is isometrically covered by a fixed irreducible symmetric space $\Omega$ of noncompact type, and that each leaf of $N$ has nonpositive sectional curvature. Find a homotopy invariant for $f$ whose non-triviality implies the existence of an isometric totally geodesic immersion of $\Omega$ into some or most of the leaves of $N$.

A variation of this problem can be obtained by replacing $N$ above with a Riemannian manifold.

Problem B. Let $M$ be compact smooth manifold with a smooth leafwise Riemannian metric, $N$ a compact Riemannian manifold with nonpositive sectional curvature and $f: M \rightarrow N$ a smooth map. Assume that each leaf of $M$ is isometrically covered by a fixed irreducible symmetric space $\Omega$ of noncompact type. Find a homotopy invariant for $f$ whose non-triviality implies the existence of an isometric totally geodesic immersion of $\Omega \hookrightarrow N$.

The solution of Problems A and B is the main goal of this paper. Our techniques are based on the use of a homotopy invariant for maps defined over foliations, called the stretch. We also make use of extensions to foliated spaces of Bochner formulae and the theory of harmonic maps. 
1.1. Remarks on notation and conventions. For the definition of a smooth foliation we refer to Godbillon [9]. This also contains a definition of a finite invariant transverse measure on a smooth foliation. Another reference for these concepts is Moore-Schochet [17], which also shows how to build a globally defined measure on a foliated space out of a finite invariant transverse measure and suitable measures on the leaves. The latter reference also considers the notion of foliation on metric spaces as required at some points of this work.

Given an object from differential geometry defined in terms of sections of bundles coming from the tangent bundle of a manifold, we can consider corresponding notions on a foliation by using the bundle of vectors tangent to the leaves, i.e. the involutive distribution associated to the foliation. We refer to such objects as leafwise defined on the manifold carrying the foliation. For example, a leafwise Riemannian metric on a foliated manifold (or metric space) is a section of the bundle of positive definite symmetric bilinear forms over the tangent spaces to the leaves. Another example of these constructions is given by the leafwise exterior forms and the leafwise cohomology; the latter is considered in [17], where it is called tangential cohomology.

Given a leafwise Riemannian metric $g$ on a foliated space $M$, we can consider the Riemannian measures on the leaves. These turn out to form a leafwise measure with the same transversal regularity that $g$ has, and we will denote it by $d v$. If $\mu$ is a finite invariant transverse measure, then we can consider a global measure on $M$, and integration with respect to this will be usually denoted by using $d \mu d v$. However, whenever we want to compute the measure of a subset of $M$ we will use $\tilde{\mu}$ to denote the global measure determined by the integration $d \mu d v$.

From now on, $M$ will denote a compact smooth manifold with a smooth foliation carrying a finite invariant transverse measure. We will also assume that $M$ carries a leafwise Riemannian metric $g$ for which each one of the leaves is a Riemannian manifold isometrically covered by a fixed irreducible symmetric space of non-compact type $\Omega$. In turn $\Omega$ will be always assumed to be of rank $\geq 2$ or a Cayley or quaternionic hyperbolic space.

We also consider most of the basic differential operators of Riemannian geometry along the leaves of a foliation, like the exterior differential or the Levi-Civita connection. Even though these are only defined along the leaves of a foliation we use the same standard symbols for them, since we only have occasion to consider those operators on the leaves. However, for the commonly used bundles associated to a foliation we use $\mathcal{F}$ as a subindex to emphasize its dependence on the foliation, e.g. the leafwise tangent bundle of a foliated space $M$ is denoted by $T_{\mathcal{F}} M$.

We are interested in considering maps from $M$ into a compact manifold $N$ carrying some sort of Riemannian structure. In view of Problems A and B we will consider two choices for $N$. It is either a compact Riemannian manifold or a compact manifold with a smooth foliation carrying a smooth leafwise Riemannian metric. When necessary we will specify our choice, but we will denote by the same symbol $h$ the metric for $N$, whether it is a Riemannian metric or a leafwise Riemannian metric.

1.2. Main theorems and sketch of their proofs. For Riemannian manifolds a geometric superrigidity has been developed for symmetric spaces by making use of the existence of harmonic maps which are then proved to be totally geodesic with the use of Bochner formula techniques. 
Gromov [11] has considered the existence problem of harmonic maps from a foliation with a leafwise Riemannian metric into a Riemannian manifold. His techniques are based on a study of the parabolic heat equation along the leaves of a foliation, whose solution for all time is guaranteed by the work of Li and Tam [15]. Corlette-Zimmer [3] pointed out that most of the basic results of Gromov [11] can be extended easily to leaf preserving maps between foliations. However, in both setups it is not possible in general to have convergence of the heat flow as $t \rightarrow \infty$, so we do not have a ready-to-use existence theorem for harmonic maps.

On the other hand, the Bochner formula of Mok-Siu-Yeung [16] can be easily generalized to foliated manifolds, and we establish this fact in Theorem 4.3. Hence, the main difficulty in extending the arguments of geometric superrigidity for manifolds to foliations lies in the existence problem for harmonic maps.

With regard to this, it has been proved in [11] that the presence of a finite invariant transverse measure on our foliated manifold $M$ implies the existence of harmonic maps from the leaves of $M$ into Riemannian manifolds, whenever we start a heat flow with a map of bounded energy density. In subsection 4.2 we use our foliated Bochner formula and its corollaries to extend this result of [11] and prove the existence of totally geodesic maps from the leaves of $M$ into either $N$ or the leaves of $N$ (according to whether $N$ is a Riemannian manifold or a foliated manifold with a leafwise Riemannian metric), whenever we start a heat flow with a smooth map $f: M \rightarrow N$ (leaf preserving when $N$ is a foliated space). Our relevant result here is Theorem 4.6.

However we need the totally geodesic maps to be non-constant to solve Problems $\mathrm{A}$ and $\mathrm{B}$, and hence we need to impose some additional condition to achieve this. In [11] the notion of stretch of a map is introduced as a straightforward generalization to foliations of what is called in [4] the intersection of a map. In section 2 we consider the stretch for both maps between foliations and maps from foliations to Riemannian manifolds. For a map $f: M \rightarrow N$ the stretch is a non-negative real number denoted by stre $(f)$ which, as remarked in [4] and [11], is an invariant of leaf preserving homotopies. We further prove that it is invariant under a measurable heat flow (see subsections 2.2 and 3.3).

On the other hand, we consider the stretch of a map $f$ as above to be non-trivial when it is positive. To better understand this condition we remark that, for the case of $N$ a Riemannian manifold, it turns out that stre $(f)$ is positive if $f$ maps a large number of leaves of $M$ into $N$ so that their lifts to the universal cover of $N$ grow to infinity at least as fast as the growth of the geodesics in the universal covers of the corresponding leaves in $M$ (see Definition 2.7). Hence the condition stre $(f)>0$ ensures a certain proper behavior of $f$ at the level of universal covers. When we apply this to the identity map we obtain a condition that imposes a restriction on the way the leaves are wrapped around a compact manifold.

Then an inequality between the stretch and the total energy of a map (see Proposition 2.9) is used to show that the totally geodesic maps from the leaves of $M$ into either $N$ or the leaves of $N$ are non-constant whenever the initial map $f: M \rightarrow N$ for the heat flow equation has positive stretch. This allows us to prove that the stretch of a map is a homotopy invariant that provides solutions to Problems A and B (see Theorems 4.8 and 4.14).

Finally we develop conditions for the stretch to be positive, and obtain as corollaries the following applications to both the geometry of foliations and Riemannian manifolds: 
Theorem 4.15. Let $M$ be a compact manifold with a smooth foliation $\mathcal{F}$, a finite invariant transverse measure and a smooth leafwise Riemannian metric $g$. Assume that each leaf is isometrically covered by an irreducible symmetric space $\Omega$ of noncompact type, which is either of rank $\geq 2$ or a Cayley or quaternionic hyperbolic space. Let $h$ be a smooth leafwise Riemannian metric on $M$ which has nonpositive sectional curvature if $\operatorname{rank}(\Omega) \geq 2$, and nonpositive complexified sectional curvature otherwise. Then $g$ and $h$ are homothetic on $\mu$-a.e. leaf of $M$.

Theorem 4.12. Let $(M, g)$ be a compact connected Riemannian manifold such that its universal cover $(\tilde{M}, g)$ has as a de Rham factor an irreducible symmetric space $\Omega$ of noncompact type which is either of rank $\geq 2$ or a Cayley or quaternionic hyperbolic space. If $h$ is a Riemannian metric on $M$ with nonpositive sectional curvature in the case of $\operatorname{rank}(\Omega) \geq 2$ and with nonpositive complexified sectional curvature otherwise, then the Riemannian universal cover of $(M, h)$ has an irreducible de Rham factor containing $\Omega$ by an isometric totally geodesic embedding. In particular, if $\operatorname{rank}(\Omega) \geq 2$ and all rank 1 factors of $(\tilde{M}, h)$ have strictly negative sectional curvature, then through every point of $(M, h)$ there is an isometric and totally geodesic immersed copy of $\Omega$.

In a personal communication, Patrick Eberlein has remarked that using [5] and [6] it is possible to show a result similar to Theorem 4.12 if we assume stronger conditions on the geometry of $(M, g)$. More specifically, Theorem 4.12 follows from [5] and [6] if we further assume that the Riemannian universal cover of $(M, g)$ isometrically splits as $(\tilde{M}, g)=\left(\Omega, g_{1}\right) \times\left(Y, g_{2}\right)$, so that either $\left(Y, g_{2}\right)$ has discrete isometry group or it has nonpositive sectional curvature and no Euclidean factors. Eberlein's techniques are different from those considered here, and none of these restrictions on $Y$ are required for Theorem 4.12.

In section 4 we develop several other results that show how positivity of the stretch imply rigidity type properties for the geometry of foliations $M$ as described before.

\section{Stretch of Maps and Foliations}

2.1. Preliminary definitions and properties. We start this subsection with the following definition which is motivated by [4].

Definition 2.1. Let $(X, g)$ and $(Y, h)$ be complete Riemannian manifolds and $f: X \rightarrow Y$ a smooth map, and let $S X$ be the unit tangent bundle of $X$. We define a function $\phi: \mathbb{R} \times S X \rightarrow \mathbb{R}$ as follows. For $v \in S X$ and $t \in \mathbb{R}$, let $\gamma_{t}$ be the geodesic with initial velocity vector $v$ and restricted to either $[0, t]$ or $[t, 0]$. Then $\phi_{t}(v)=\phi(t, v)=$ minimum of the lengths of paths in $Y$ homotopic with fixed endpoints to $f\left(\gamma_{t}\right)$.

Remark 2.2. Note that in Definition 2.1 the minimum is attained at some geodesic $\alpha$ in $Y$, joining the endpoints of $f\left(\gamma_{t}\right)$, having a lift to the universal cover of $Y$ with the same endpoints as some lift of $f\left(\gamma_{t}\right)$. If $Y$ has no conjugate points, then $\alpha$ is the image onto $Y$ of the unique geodesic in the universal cover of $Y$ joining the endpoints of a fixed lift of $f\left(\gamma_{t}\right)$.

The function $\phi_{t}$ in Definition 2.1 seeks to measure the non-triviality of the map $f$ with respect to the geometry of the Riemannian manifolds $X$ and $Y$.

In [4] the following property of $\phi_{t}$ is proved. 
Lemma 2.3. For $X, Y$ and $f$ as above,

$$
\phi_{t+t^{\prime}}(v) \leq \phi_{t^{\prime}}\left(g_{t} v\right)+\phi_{t}(v),
$$

where $t, t^{\prime} \in \mathbb{R}, v \in S X$ and $g_{t}: S X \rightarrow S X$ is the geodesic flow.

The following definition provides invariants that allow to use the function $\phi_{t}$ to understand some of the geometric features of a smooth map between Riemannian manifolds.

Definition 2.4. Let $(X, g)$ and $(Y, h)$ be complete Riemannian manifolds, and for a smooth map $f: X \rightarrow Y$ consider $\phi_{t}$ as in Definition 2.1. We define the pointwise stretch of $f$ as the function on $S X$ given by

$$
\text { p-stre }(f)(v)=\liminf _{t \rightarrow \infty} \frac{\phi_{t}(v)}{t} .
$$

If $X$ is compact, then we define the stretch of $f$ by

$$
\operatorname{stre}(f)=\inf _{t \in \mathbb{R}^{+}} \int_{S X} \frac{\phi_{t}(v)}{t} d L(v)=\lim _{t \rightarrow \infty} \int_{S X} \frac{\phi_{t}(v)}{t} d L(v),
$$

where $d L$ denotes the Liouville measure on $S X$ obtained from $g$, i.e. the Riemannian measure on $S X$ associated to the natural Riemannian structure on $S X$ coming from $g$.

Remark 2.5. The last identity in the definition of stretch follows from the invariance of $d L$ under $g_{t}$ and by Lemma 2.3. Furthermore for $X$ compact, by the subadditive ergodic theorem, the limit

$$
\lim _{t \rightarrow \infty} \frac{\phi_{t}(v)}{t}
$$

exists for $L$-a.e. $v \in S X$, and we have

$$
\operatorname{stre}(f)=\int_{S X} \mathrm{p}-\operatorname{stre}(f)(v) d L(v) .
$$

As an example of how the stretch measures the non-triviality of a map we have the following:

Proposition 2.6. If $X$ and $Y$ are compact Riemannian manifolds, $f: X \rightarrow Y$ is a smooth (not necessarily Riemannian) covering and $X$ has no conjugate points, then there is a constant $C>0$ such that $\mathrm{p}$-stre $(f) \geq C$. In particular, $\operatorname{stre}(f)>0$.

Proof. Let $v \in S X, t \geq 0$ and $\gamma_{t}$ the geodesic with initial velocity vector $v$ restricted to $[0, t]$. Let $g$ and $h$ be the Riemannian metrics of $X$ and $Y$, respectively. Denote by $\alpha$ a geodesic in $Y$ homotopic with fixed endpoints to $f\left(\gamma_{t}\right)$ satisfying:

$$
\phi_{t}(v)=\operatorname{length}_{(Y, h)}(\alpha) .
$$

Since $f$ is a covering, there is a path $\alpha_{0}$ in $X$ homotopic with fixed endpoints to $\gamma_{t}$ so that $\alpha=f\left(\alpha_{0}\right)$. Being $(X, g)$ without conjugate points, the lifts of $\gamma_{t}$ to the universal cover of $X$ realize distance between their endpoints, and by lifting the fixed endpoints homotopy between $\gamma_{t}$ and $\alpha_{0}$ we observe that

$$
\operatorname{length}_{(X, g)}\left(\alpha_{0}\right) \geq \operatorname{length}_{(X, g)}\left(\gamma_{t}\right)=t .
$$

Since $X$ is compact $g$ and $f^{*} h$ are quasi-isometric, so there is a constant $C>0$ such that

$$
\operatorname{length}_{\left(X, f^{*} h\right)}\left(\alpha_{0}\right) \geq C \text { length }_{(X, g)}\left(\alpha_{0}\right) .
$$


But since $f$ is a covering and $\alpha=f\left(\alpha_{0}\right)$, we have

$$
\operatorname{length}_{(Y, h)}(\alpha)=\operatorname{length}_{\left(X, f^{*} h\right)}\left(\alpha_{0}\right) .
$$

From all of the above inequalities we obtain

$$
\phi_{t}(v) \geq C t
$$

from which the conclusion clearly follows.

As shown in [4], the notion of stretch for compact Riemannian manifolds can be used to understand their geometry. Here, we will follow Gromov [11] and use it to understand the leafwise geometry of a foliation.

In the rest of the subsection we will assume, as stated in subsection 1.1, that $M$ is a compact manifold with a smooth foliation $\mathcal{F}$ carrying a leafwise Riemannian metric $g$ and a finite invariant transverse measure $\mu$, and we will make use of the previous remarks.

Denote by $S_{\mathcal{F}} M$ the leafwise unit tangent bundle of $M$, i.e. the smooth bundle over $M$ whose fibers are the tangent vectors to the leaves of $\mathcal{F}$ which are unitary for $g . S_{\mathcal{F}} M$ has a natural foliation such that the canonical projection $p: S_{\mathcal{F}} M \rightarrow$ $M$ is a smooth leaf preserving map, and so that the inverse image of a leaf is a leaf. Moreover, $p$ maps local transversals of $S_{\mathcal{F}} M$ diffeomorphically into local transversals of $M$, and since $M$ has a finite invariant transverse measure $\mu$ then $S_{\mathcal{F}} M$ inherits $\mu$ as a finite invariant transverse measure. Furthermore, any such $\mu$ on $S_{\mathcal{F}} M$ and the Liouville measures on the unit tangent bundles of the leaves of $M$ (obtained from the leafwise Riemannian structure $g$ ) add up to a finite measure on $S_{\mathcal{F}} M$, which we denote by $\mu_{L}$. For $g_{t}$ the leafwise geodesic flow on $S_{\mathcal{F}} M$, it is clear that $\mu_{L}$ is $g_{t}$-invariant.

Definition 2.7. Let $M$ be a compact manifold with a smooth foliation $\mathcal{F}$ carrying a leafwise Riemannian metric $g$, let $N$ be a smooth manifold and $f: M \rightarrow N$ a measurable leafwise smooth map. Assume that either one of the following holds:

(a) $N$ carries a smooth complete Riemannian metric $h$ and $f$ is leafwise smooth, or

(b) $N$ has a smooth foliation carrying a smooth leafwise Riemannian metric $h$ which is complete in every leaf and $f$ is a leaf preserving and leafwise smooth map.

We define a map $\phi: \mathbb{R} \times S_{\mathcal{F}} M \rightarrow \mathbb{R}$ by restricting $f$ to each leaf of $M$ and applying Definition 2.1. Then the pointwise stretch of $f$ is the function on $S_{\mathcal{F}} M$ defined by

$$
\text { p-stre }(f)(v)=\liminf _{t \rightarrow \infty} \frac{\phi_{t}(v)}{t} .
$$

If $M$ has a finite invariant transverse measure $\mu$, then the stretch of $f$ is defined by

$$
\operatorname{stre}(f)=\inf _{t \in \mathbb{R}^{+}} \int_{S_{\mathcal{F}} M} \frac{\phi_{t}(v)}{t} d \mu_{L}(v)=\lim _{t \rightarrow \infty} \int_{S_{\mathcal{F}} M} \frac{\phi_{t}(v)}{t} d \mu_{L}(v) .
$$

Remark 2.8. As in Remark 2.5, the last identity follows from Lemma 2.3. Furthermore, the limit

$$
\lim _{t \rightarrow \infty} \frac{\phi_{t}(v)}{t}
$$


exists for $\mu_{L}$-a.e. $v \in S_{\mathcal{F}} M$, and we have

$$
\operatorname{stre}(f)=\int_{S_{\mathcal{F}} M} \mathrm{p}-\operatorname{stre}(f)(v) d \mu_{L}(v) .
$$

The main feature we want to exploit from the stretch of a map is its ability to control the total energy of the map. More specifically, we have the following result which generalizes an inequality proved in [4].

Proposition 2.9. Let $M, N$ and $f: M \rightarrow N$ be as in Definition 2.7. Assume that $M$ has a finite invariant transverse measure $\mu$ and that $f$ is a map with finite total energy, i.e.

$$
E(f)=\frac{1}{2} \int_{M}|d f|^{2} d \mu d v<\infty
$$

Then we have

$$
\begin{aligned}
& E(f) \geq \frac{n}{2 C_{n-1}^{2} \tilde{\mu}(M)} \operatorname{stre}(f)^{2}, \\
& \int_{M}|d f| d \mu d v \geq \frac{1}{C_{n-1}} \operatorname{stre}(f),
\end{aligned}
$$

where $n$ is the dimension of the leaves of $M, C_{n-1}$ is the volume of the unit sphere in $\mathbb{R}^{n}$ and $\tilde{\mu}$ is the global measure on $M$ defined by $\mu$ and the leafwise Riemannian volumes on $M$. Moreover, the inequality (a) is strict unless $f$ is leafwise a Riemannian homothety and the image under $f$ of every geodesic in a leaf of $M$ is homotopy minimizing in $N$.

Proof. It is a straightforward computation that follows the steps found in [4].

2.2. Homotopy invariance of the stretch. For the main applications of Proposition 2.9 we will show that the stretch of a map is invariant under certain homotopies. Here we have the following easy generalization of a result from [4].

Proposition 2.10. Let $M, N$ and $f: M \rightarrow N$ be as in Definition 2.7. Assume that $f$ is continuous and that $M$ carries a finite invariant transverse measure $\mu$. If $f_{t}$, with $0 \leq t \leq 1$, is a continuous homotopy of $f$ with $f=f_{0}$, which is leaf preserving in the case of $N$ a foliated manifold, then $\operatorname{stre}(f)=\operatorname{stre}\left(f_{1}\right)$.

Proof. Since $M$ is compact, there is a constant $C$ such that for every $m \in M$ the path $f_{s}(m), s \in[0,1]$, is homotopic with fixed endpoints to a path in $N$ whose length is $\leq C$. In the case of $N$ a foliated manifold we are assuming that the homotopy takes place within a leaf.

Hence, if $\phi_{t}$ and $\psi_{t}$ denote the functions as given in Definition 2.7 for $f$ and $f_{1}$, respectively, then it is easy to check that

$$
\phi_{t}(v) \leq \psi_{t}(v)+2 C
$$

for all $v$. Then dividing by $t$ and taking the limit as $t \rightarrow \infty$ we obtain $\operatorname{stre}(f) \leq$ stre $\left(f_{1}\right)$, and the reverse inequality is proved with the same argument.

In subsection 3.3 we will extend this result by proving that the stretch is invariant under a measurable leafwise heat flow. 
2.3. Positively stretched foliations. From what we have already discussed it should be clear that a map with positive stretch has some geometric non-triviality. Because of this, for the geometric rigidity applications it will be useful to have a criterion to ensure that a map has positive stretch. Such a result would be more useful if one could make it depend as much as possible on the spaces involved. In order to achieve this we introduce the notion of positive stretch for compact foliations.

Definition 2.11. Let $M$ be a compact manifold with a smooth foliation $\mathcal{F}$ and a leafwise Riemannian metric $g$. Assume that $h$ is a Riemannian metric on $M$. Then by using the identity map $i d:(M, \mathcal{F}, g) \rightarrow(M, h)$ we define the pointwise stretch of $M$ as the function on $S_{\mathcal{F}} M$ given by

$$
\mathrm{p} \text {-stre }(M, h ; \mathcal{F}, g)=\text { p-stre }(i d) .
$$

Similarly, if $M$ carries a finite invariant transverse measure $\mu$, then the stretch of $M$ is defined by

$$
\operatorname{stre}(M, h ; \mathcal{F}, g)=\operatorname{stre}(i d) .
$$

We say that $M$ together with the above given structure is positively stretched if $\operatorname{stre}(M, h ; \mathcal{F}, g)>0$.

By compactness, there is certain independence of the properties of the stretch of a foliation with respect to the choice of $h$.

Proposition 2.12. Let $M$ be a compact manifold with a smooth foliation $\mathcal{F}$, a leafwise Riemannian metric $g$ and a finite invariant transverse measure $\mu$. If $h_{1}$ and $h_{2}$ are Riemannian metrics on $M$, then there exist constants $a, b>0$ such that

$$
a \operatorname{stre}\left(M, h_{1} ; \mathcal{F}, g\right) \leq \operatorname{stre}\left(M, h_{2} ; \mathcal{F}, g\right) \leq b \operatorname{stre}\left(M, h_{1} ; \mathcal{F}, g\right) .
$$

Proof. Let $\phi_{t}$ and $\psi_{t}$ be the functions as in Definition 2.7 corresponding to the identity maps $(M, \mathcal{F}, g) \rightarrow\left(M, h_{1}\right)$ and $(M, \mathcal{F}, g) \rightarrow\left(M, h_{2}\right)$, respectively. For $v \in S_{\mathcal{F}} M$ and $t \geq 0$, let $\gamma_{t}$ be the $g$-geodesic with initial velocity vector $v$ restricted to $[0, t]$. Denote by $\alpha_{1}$ and $\alpha_{2}$ the geodesics in $\left(M, h_{1}\right)$ and $\left(M, h_{2}\right)$, respectively, that are homotopic with fixed endpoints to $\gamma_{t}$ and satisfy $\phi_{t}(v)=\operatorname{length}_{h_{1}}\left(\alpha_{1}\right)$ and $\psi_{t}(v)=$ length $_{h_{2}}\left(\alpha_{2}\right)$.

If $\tilde{\gamma}_{t}$ is a lift of $\gamma_{t}$ to the universal cover of $M$ and $\tilde{\alpha}_{1}, \tilde{\alpha}_{2}$ are lifts of $\alpha_{1}, \alpha_{2}$ with the same endpoints, then by the choice of $\alpha_{1}$ the geodesic $\tilde{\alpha}_{1}$ realizes the $h_{1}$ distance between its endpoints, and a similar condition holds for $\tilde{\alpha}_{2}$. Since $h_{1}$ and $h_{2}$ are quasi-isometric when lifted to the universal cover of $M$, it follows that there is a constant $c$ depending on the metrics only such that

$$
\operatorname{length}_{h_{1}}\left(\alpha_{1}\right)=\operatorname{length}_{h_{1}}\left(\tilde{\alpha}_{1}\right) \geq c \operatorname{length}_{h_{2}}\left(\tilde{\alpha}_{2}\right)=\operatorname{length}_{h_{2}}\left(\alpha_{2}\right) .
$$

Hence it follows that $\phi_{t}(v) \geq c \psi_{t}(v)$, and this implies

$$
\operatorname{stre}\left(M, h_{1} ; \mathcal{F}, g\right) \geq c \operatorname{stre}\left(M, h_{2} ; \mathcal{F}, g\right) .
$$

Remark 2.13. From the above proposition it follows that:

$$
\operatorname{stre}\left(M, h_{1} ; \mathcal{F}, g\right)>0 \Leftrightarrow \operatorname{stre}\left(M, h_{2} ; \mathcal{F}, g\right)>0 .
$$

When this holds we write $\operatorname{stre}(M, \mathcal{F}, g)>0$, and we say $(M, \mathcal{F}, g)$, or simply that $M$, is positively stretched. 
The condition of a foliated manifold being positively stretched can be used to prove that maps with certain properties have positive stretch. For the following example, we recall that a submanifold $Y$ of a Riemannian manifold $(N, h)$ is said to have bounded distortion if the distance functions $\left.d_{(N, h)}\right|_{Y}$ and $d_{\left(Y,\left.h\right|_{Y}\right)}$ are quasiisometric.

Proposition 2.14. Let $M$ be a compact connected manifold with a smooth foliation $\mathcal{F}$, a leafwise Riemannian metric $g$ and a finite invariant transverse measure $\mu$. Let $(N, h)$ be a complete Riemannian manifold and $f: M \rightarrow N$ a smooth map. Assume that the following hold:

(a) $f$ is an immersion and $Y=f(M)$ is a manifold,

(b) there is a lift $\tilde{f}: \tilde{M} \rightarrow \tilde{N}$ to universal covers, such that $\tilde{f}(\tilde{M})=\tilde{Y}$ is a simply connected bounded distortion submanifold of $\tilde{N}$.

Then there is a constant $C>0$ such that

$$
\operatorname{stre}(f) \geq C \operatorname{stre}\left(M, f^{*} h ; \mathcal{F}, g\right) .
$$

In particular, if $(M, \mathcal{F}, g)$ is positively stretched, then $\operatorname{stre}(f)>0$.

Proof. Let $\phi_{t}$ and $\tilde{\phi}_{t}$ be the functions given in Definition 2.7 corresponding to $f: M \rightarrow N$ and $i d:(M, \mathcal{F}, g) \rightarrow\left(M, f^{*} h\right)$, respectively. Fix $v \in S_{\mathcal{F}} M$ and $t \geq 0$, and let $\gamma_{t}$ be the geodesic determined by $v$ restricted to $[0, t]$.

Denote by $\alpha$ a geodesic in $(Y, h)$ of minimun length among all paths homotopic in $Y$, with fixed endpoints, to $f\left(\gamma_{t}\right)$. Also, denote with $\beta$ the geodesic in $(N, h)$ of minimum length among all paths homotopic in $N$, with fixed endpoints, to $f\left(\gamma_{t}\right)$. Then by definition we have

$$
\phi_{t}(v)=\operatorname{length}_{(N, h)}(\beta) .
$$

If $\delta_{t}$ is a lift of $f\left(\gamma_{t}\right)$ to $\tilde{Y}$, then, since $\tilde{N}$ and $\tilde{Y}$ are simply connected,

$$
\begin{aligned}
\operatorname{length}_{(N, h)}(\beta) & =d_{(\tilde{N}, h)}\left(\delta_{t}(0), \delta_{t}(t)\right), \\
\operatorname{length}_{(Y, h)}(\alpha) & =d_{(\tilde{Y}, h)}\left(\delta_{t}(0), \delta_{t}(t)\right) .
\end{aligned}
$$

Hence, by the bounded distortion condition on $\tilde{Y}$, there is a constant $C>0$, independent of $t$ and $v$, such that

$$
\text { length }_{(N, h)}(\beta) \geq C \text { length }_{(Y, h)}(\alpha) \text {. }
$$

By compactness $f: M \rightarrow Y$ is a covering, and since $\alpha$ is homotopic with fixed endpoints to $f\left(\gamma_{t}\right)$, there is a path $\alpha_{0}$ in $M$, homotopic with fixed endpoints to $\gamma_{t}$, such that $f\left(\alpha_{0}\right)=\alpha$. In particular,

$$
\operatorname{length}_{(Y, h)}(\alpha)=\operatorname{length}_{\left(M, f^{*} h\right)}\left(\alpha_{0}\right) .
$$

On the other hand, since $\tilde{\phi}_{t}(v)$ is the minimum of the lengths in $\left(M, f^{*} h\right)$ of paths homotopic in $M$ with fixed endpoints to $\gamma_{t}$, we have

$$
\operatorname{length}_{\left(M, f^{*} h\right)}\left(\alpha_{0}\right) \geq \tilde{\phi}_{t}(v) .
$$

And then collecting the relations obtained we find that

$$
\phi_{t}(v) \geq C \tilde{\phi}_{t}(v),
$$

which after integrating yields

$$
\operatorname{stre}(f) \geq C \operatorname{stre}\left(M, f^{*} h ; \mathcal{F}, g\right) .
$$


As an immediate consequence of this result we have:

Corollary 2.15. With $(M, \mathcal{F}, g)$ and $(N, h)$ as above, if $f: M \rightarrow N$ is a smooth covering, then

$$
\operatorname{stre}(f) \geq C \operatorname{stre}\left(M, f^{*} h ; \mathcal{F}, g\right)
$$

for some constant $C>0$. In particular, if $(M, \mathcal{F}, g)$ is positively stretched, then $\operatorname{stre}(f)>0$.

We now prove a sufficient condition for a foliation to be positively stretched.

Proposition 2.16. Let $M$ be a compact manifold with a smooth foliation $\mathcal{F}$ and a finite invariant transverse measure $\mu$. Assume $h$ is a Riemannian metric on $M$ satisfying:

(a) in the universal cover $(\tilde{M}, h)$ the induced foliation $\tilde{\mathcal{F}}$ has leaves with uniform bounded distortion and a.e. leaf is simply connected,

(b) $\left.h\right|_{\mathcal{F}}$ is a leafwise Riemannian metric on $(M, \mathcal{F})$ without conjugate points.

Then

$$
\operatorname{stre}\left(M, h ; \mathcal{F},\left.h\right|_{\mathcal{F}}\right)>0 .
$$

In particular, $\left(M, \mathcal{F},\left.h\right|_{\mathcal{F}}\right)$ is positively stretched.

Proof. Let $\phi_{t}$ be the function given in Definition 2.7 corresponding to the identity map id: $\left(M, \mathcal{F},\left.h\right|_{\mathcal{F}}\right) \rightarrow(M, h)$. Fix $t \geq 0$ and $v \in S_{\mathcal{F}} M$ so that the leaf $\mathcal{L}$ containing $p(v)$ is covered by a simply connected leaf $\tilde{\mathcal{L}}$ in $\tilde{M}$, and let $\gamma_{t}$ be the

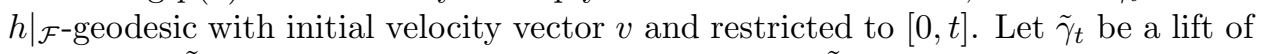
$\gamma_{t}$ lying in $\tilde{\mathcal{L}}$. Since $\left.h\right|_{\mathcal{F}}$ has no conjugate points and $\tilde{\mathcal{L}}$ is simply connected,

$$
d_{\tilde{\mathcal{L}}}\left(\tilde{\gamma}_{t}(0), \tilde{\gamma}_{t}(t)\right)=\operatorname{length}\left(\tilde{\gamma}_{t}\right)=\operatorname{length}\left(\gamma_{t}\right)=t .
$$

Let $\alpha$ be an $h$-geodesic in $M$ with minimum length among all paths homotopic to $\gamma_{t}$ in $M$ with fixed endpoints. Then by definition

$$
\phi_{t}(v)=\operatorname{length}(\alpha) \text {. }
$$

Since $\alpha$ is homotopic with fixed endpoints to $\gamma_{t}$, it has a lift, say $\tilde{\alpha}$, to $\tilde{M}$ with the same endpoints of $\tilde{\gamma}_{t}$. By the choice of $\alpha$ the lift $\tilde{\alpha}$ minimizes the distance in $(\tilde{M}, h)$ between its endpoints, which are those of $\tilde{\gamma}_{t}$, and hence

$$
\operatorname{length}(\alpha)=\operatorname{length}(\tilde{\alpha})=d_{\tilde{M}}\left(\tilde{\gamma}_{t}(0), \tilde{\gamma}_{t}(t)\right)
$$

Since the foliation $\tilde{\mathcal{F}}$ has leaves with uniform bounded distortion, there is a constant $C>0$, depending only on $h$ and $\tilde{\mathcal{F}}$, such that

$$
d_{\tilde{M}}\left(\tilde{\gamma}_{t}(0), \tilde{\gamma}_{t}(t)\right) \geq C d_{\tilde{\mathcal{L}}}\left(\tilde{\gamma}_{t}(0), \tilde{\gamma}_{t}(t)\right) .
$$

Since $\left.h\right|_{\mathcal{L}}$ has no conjugate points, we have

$$
d_{\tilde{\mathcal{L}}}\left(\tilde{\gamma}_{t}(0), \tilde{\gamma}_{t}(t)\right)=t
$$

Collecting the above relations we find that $\phi_{t}(v) \geq C t$, and hence

$$
\operatorname{stre}\left(M, h ; \mathcal{F},\left.h\right|_{\mathcal{F}}\right)>C \mu_{L}\left(S_{\mathcal{F}} M\right)>0 .
$$

As a consequence we obtain the following result: 
Proposition 2.17. If $(M, h)$ is a compact manifold without conjugate points and $\mathcal{F}$ is a totally geodesic foliation of $M$ carrying a finite invariant transverse measure, then

$$
\operatorname{stre}\left(M, h ; \mathcal{F},\left.h\right|_{\mathcal{F}}\right)>0 .
$$

Proof. Let $\tilde{M}$ be the universal cover of $M$. Since $(\tilde{M}, h)$ has no conjugate points and the induced foliation $\tilde{\mathcal{F}}$ is totally geodesic, the leaves are isometrically (in the sense of metric spaces) immersed in $\tilde{M}$. Also, being totally geodesic, the leaves of $\tilde{\mathcal{F}}$ are simply connected since $\tilde{M}$ is simply connected. Finally, since $\mathcal{F}$ is totally geodesic the leafwise metric $\left.h\right|_{\mathcal{F}}$ has no conjugate points, so Proposition 2.16 gives the conclusion.

Also as a corollary we have:

Proposition 2.18. Let $(M, h)$ be a compact connected Riemannian manifold whose universal cover $\tilde{M}$ has a de Rham factor $\left(\Omega, g_{0}\right)$ without conjugate points. Assume that the foliation in $\tilde{M}$ defined by $\Omega$ induces a foliation $\mathcal{F}$, and let $g$ be the leafwise Riemannian metric induced by $g_{0}$. Then $(M, \mathcal{F}, g)$ is positively stretched.

Proof. In the universal cover $\tilde{M}$ of $M$ the lifted foliation $\tilde{\mathcal{F}}$ has leaves given by $\Omega$, so they are clearly simply connected and isometrically immersed (as metric spaces) in $\tilde{M}$. Since $g_{0}$ has no conjugate points, the same holds true for $g$. Hence, the conclusion follows from Proposition 2.16.

Because of the applications we are seeking we will consider foliations by symmetric spaces of noncompact type, which will turn out to be positively stretched.

Let $H$ be a connected centerless semisimple Lie group of noncompact type, $G \subset$ $H$ a connected closed semisimple subgroup of noncompact type, $\Gamma \subset H$ a torsionless cocompact lattice and $K \subset G$ a maximal compact subgroup. Then $M=\Gamma \backslash H / K$ is a compact manifold with a foliation $\mathcal{F}$ induced by the right $G$-orbits in $H$, and the leaves are smoothly covered by $\Omega=G / K$. It is easy to see that $\mathcal{F}$ has a finite invariant transverse measure $\mu$. For this space the following is satisfied:

Lemma 2.19. Let $H, G$ and $\Gamma$ be as above. Then:

(a) Any $H$-invariant metric $h$ on $H / K$ induces a smooth leafwise Riemannian metric $g=\left.h\right|_{\mathcal{F}}$ on $\Gamma \backslash H / K$ with leaves locally isometric to the symmetric space $\Omega=G / K$.

(b) For any two choices of $K$ and $h$ as in (a), the resulting leafwise Riemannian metrics are homothetic on the irreducible factors of the universal covers of the leaves, with homothety constants independent of the leaves. In particular, for $\left(\Gamma \backslash H / K, h ; \mathcal{F},\left.h\right|_{\mathcal{F}}\right)$ the property of being positively stretched is independent of the choice of the $H$-invariant metric $h$ and the maximal compact subgroup $K$ of $G$.

Proof. Let $h$ and $g$ be as in (a). The right $G$-orbit in $H$ passing through $x$ maps in the space $H / K$ onto the leaf $x G / K$. In particular, the leaf through $e$ in $H / K$ is $G / K$ contained by the natural inclusion $G / K \hookrightarrow H / K$. And since the metric $h$ is $H$-invariant, the restriction of $g$ to the leaf $G / K$ is $G$-invariant and defines a symmetric space. 
On the other hand, the leaf $x G / K$ passing through $x K$ is diffeomorphically mapped onto $G / K$ by the map

$$
\begin{aligned}
& H / K \rightarrow H / K, \\
& y K \mapsto x^{-1} y K,
\end{aligned}
$$

which is a global isometry of $(H / K, h)$, and then $x G / K$ is also locally symmetric.

For fixed $K$, any two $H$-invariant metrics on $H / K$ are symmetric when restricted to $G / K$ and hence homothetic on irreducible factors. By the $H$-invariance this homothety extends to all the foliation, with constants independent of the leaves.

Now let $K$ and $K_{0}$ be maximal compact subgroups of $G$. Then there exists $x \in G$ such that $K_{0}=x^{-1} K x$ and the map

$$
\begin{aligned}
H / K & \rightarrow H / K_{0}, \\
y K & \mapsto y x K_{0},
\end{aligned}
$$

is an $H$-invariant diffeomorphism preserving the foliation induced by the right $G$ orbits in $H$. This map defines a $G$-invariant diffeomorphism between the symmetric spaces $G / K$ and $G / K_{0}$, and hence (for any choice of metrics as in (a) associated to $K$ and $\left.K_{0}\right)$ a homothety on irreducible factors. By $H$-invariance this homothety on irreducible factors extends to the whole foliation with homothety constants independent of the leaves.

Concerning the spaces described above, we have:

Theorem 2.20. Let $H, G$ and $\Gamma$ be as above. Then for $\mathcal{F}$ the foliation in $\Gamma \backslash H / K$ induced by the right $G$-orbits in $H$ we have:

(a) There is a maximal compact subgroup $K$ of $G$ and an $H$-invariant metric $h$ on $H / K$ so that $\mathcal{F}$ is totally geodesic in $(\Gamma \backslash H / K, h)$ and

$$
\operatorname{stre}\left(\Gamma \backslash H / K, h ; \mathcal{F},\left.h\right|_{\mathcal{F}}\right)=\operatorname{vol}\left(S_{\mathcal{F}} \Gamma \backslash H / K\right) \text {. }
$$

(b) For any maximal compact subgroup $K$ of $G$ and any $H$-invariant metric $h$ in $\Gamma \backslash H / K$,

$$
\operatorname{stre}\left(\Gamma \backslash H / K, h ; \mathcal{F},\left.h\right|_{\mathcal{F}}\right)>0 .
$$

Proof. The inclusion $G \subset H$ induces an inclusion $\mathfrak{g} \subset \mathfrak{h}$ of their Lie algebras. Since both $\mathfrak{g}$ and $\mathfrak{h}$ are semisimple by [19], $\mathfrak{g}$ is canonically embedded in $\mathfrak{h}$. In other words, there is a Cartan involution $\theta$ of $\mathfrak{h}$ such that $\theta(\mathfrak{g})=\mathfrak{g}$, and hence $\left.\theta\right|_{\mathfrak{g}}$ is a Cartan involution (see page 261 of [19]). Let $\mathfrak{h}=\mathfrak{l}+\mathfrak{n}$ and $\mathfrak{g}=\mathfrak{k}+\mathfrak{m}$ be the associated Cartan decompositions, where $\mathfrak{l}$ and $\mathfrak{k}$ are the maximal compact subalgebras. By the choice of the involutions we have $\mathfrak{k} \subset \mathfrak{l}$ and $\mathfrak{m} \subset \mathfrak{n}$. Let $K \subset G$ and $L \subset H$ be maximal compact subgroups with $\operatorname{Lie}(K)=\mathfrak{k}$ and $\operatorname{Lie}(L)=\mathfrak{l}$.

Since $\mathfrak{l}$ and $\mathfrak{n}$ are $\operatorname{Ad}_{H}(L)$-invariant, we can choose an $\operatorname{Ad}_{H}(L)$-invariant inner product on $\mathfrak{h}$, say $\langle\cdot, \cdot\rangle$, such that $\mathfrak{l} \perp \mathfrak{n}$. Then for the restriction of $\langle\cdot, \cdot\rangle$ to $\mathfrak{g}$ we have $\mathfrak{k} \perp \mathfrak{m}$.

Consider the left $H$-invariant Riemannian metric on $H$ determined by $\langle\cdot, \cdot\rangle$ at $T_{e} H=\mathfrak{h}$, which we will denote by the same symbol. $\operatorname{By} \operatorname{Ad}_{H}(L)$-invariance the Riemannian metric $\langle\cdot, \cdot\rangle$ is also right $L$-invariant.

Let $h$ be the $H$-invariant Riemannian metric on $H / K$ induced by $\langle\cdot, \cdot\rangle$. We will prove that the foliation in $H / K$ coming from the right $G$-orbits in $H$ is totally geodesic with respect to $h$. By $H$-homogeneity it is enough to check that the natural inclusion $G / K \hookrightarrow(H / K, h)$ is totally geodesic. 
By right $L$-invariance of $\langle\cdot, \cdot\rangle$ in $H$, this metric induces $H$-invariant Riemannian metrics on $H / K$ and $H / L$ such that the natural projections $H \rightarrow H / K$ and $H \rightarrow$ $H / L$ are Riemannian submersions. Let $\pi: H / K \rightarrow H / L$ be the natural projection; then, since the diagram

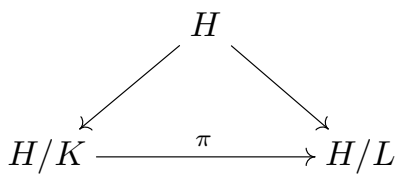

commutes, it is easy to check that $\pi$ is a Riemannian submersion.

The metric on $H$ induces a left $G$-invariant and right $K$-invariant Riemannian metric on $G$, such that $G \rightarrow G / K$ is a Riemannian submersion and $G / K \hookrightarrow H / K$ is an isometric immersion. On the other hand, since $K=G \cap L$ we have the following commutative diagram of natural maps:



where the vertical maps are Riemannian submersions and $G \rightarrow H$ is an isometric immersion. Taking differentials at $e$ we get the following commutative diagram:

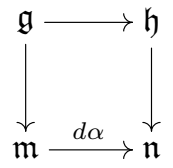

where the vertical maps are orthogonal projections associated to the Cartan decompositions. Hence, $d \alpha$ is the natural inclusion and an isometric immersion. Then, by the $G$-invariance of the corresponding diagram with Lie groups, the map $\alpha$ is an isometric immersion. Furthermore, since $H / L$ is symmetric and $\mathfrak{g}$ is $\theta$-invariant, the map $\alpha$ is totally geodesic.

Let $v \in T_{e}(G / K) \subset T_{e}(H / K)$ with $|v|=1$, and let $\gamma$ be the geodesic in $H / K$ with initial velocity vector $v$. Since $\pi$ is a Riemannian submersion, we have

$$
1=|v|_{H / K}^{2}=|d \pi(v)|_{H / L}^{2}+\left|v_{0}\right|_{H / K}^{2},
$$

where $v_{0}$ is the vertical component of $v$ with respect to $\pi$. And since the diagram

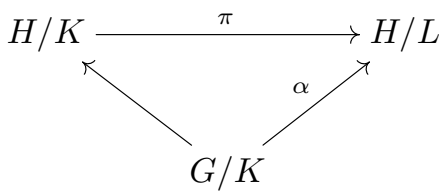

commutes, we have that

$$
|d \pi(v)|_{H / L}^{2}=|d \alpha(v)|_{H / L}^{2}=|v|_{G / K}^{2}=1,
$$

since $\alpha$ is an isometric immersion. Then the above relations show that $v_{0}=0$; in other words $v$ is $\pi$-horizontal. By [8] the geodesic $\gamma$ is $\pi$-horizontal and $\pi(\gamma)$ is a geodesic in $H / L$. The initial velocity vector of $\pi(\gamma)$ is given by

$$
(\pi \circ \gamma)^{\prime}(0)=d \pi\left(\gamma^{\prime}(0)\right)=d \pi(v)=d \alpha(v),
$$


and since the inclusion $\alpha$ is totally geodesic it follows that there is a geodesic $\gamma_{0}$ of $G / K$ such that $\alpha\left(\gamma_{0}\right)=\pi(\gamma)$. Then, by the last commutative diagram, it follows that $\pi\left(\gamma_{0}\right)=\pi(\gamma)$. On the other hand, the same argument used with $v$ proves that $\gamma_{0}$, being tangent to $G / K$, is a $\pi$-horizontal curve in $H / K$. Hence, $\gamma$ and $\gamma_{0}$ are $\pi$-horizontal lifts of the geodesic $\pi(\gamma)$ passing through the basepoint of $v$, and by uniqueness it follows that $\gamma=\gamma_{0}$. In other words, $\gamma$ is a curve in $G / K$.

From the above we conclude that $G / K \hookrightarrow H / K$ is totally geodesic at $e$, and by $G$-invariance it is totally geodesic elsewhere.

Finally we will prove that $\left(\Gamma \backslash H / K, h ; \mathcal{F},\left.h\right|_{\mathcal{F}}\right)$ is positively stretched for the above choices, and from the previous results in this subsection it will be positively stretched for any other choice of $h$ and $K$.

In fact we will prove that

$$
\operatorname{stre}\left(\Gamma \backslash H / K, h ; \mathcal{F},\left.h\right|_{\mathcal{F}}\right)=\operatorname{vol}\left(S_{\mathcal{F}} \Gamma \backslash H / K\right),
$$

and for this we will consider the universal cover of $\Gamma \backslash H / K$.

Let $\tilde{H}$ be the universal cover of $H$ and let $\tilde{G}, \tilde{K}, \tilde{L}$ be the connected closed subgroups with Lie algebras $\mathfrak{g}, \mathfrak{k}, \mathfrak{l}$, respectively. The metric $\langle\cdot, \cdot\rangle$ in $H$ lifts to a Riemannian metric on $\tilde{H}$ which is left $\tilde{H}$-invariant and right $\tilde{L}$-invariant. As before, we have a Riemannian metric $h$ on $\tilde{H} / \tilde{K}$ which is $\tilde{H}$-invariant and such that the natural map $\tilde{H} / \tilde{K} \rightarrow \Gamma \backslash H / K$ is a Riemannian universal cover.

We want to prove the following:

Claim 1. Any geodesic in $\tilde{H} / \tilde{K}$ joining two points in $\tilde{G} / \tilde{K}$ and that realizes the distance in $\tilde{H} / \tilde{K}$ between them lies entirely inside of $\tilde{G} / \tilde{K}$.

First assuming the claim, let $v \in S_{\mathcal{F}} \Gamma \backslash H / K, t \geq 0$, and let $\gamma_{t}$ be the geodesic with initial velocity vector $v$ and restricted to $[0, t]$. Let $\tilde{\gamma}_{t}$ be a lift of $\gamma_{t}$ to $\tilde{H} / \tilde{K}$. Without loss of generality, we can assume that $\tilde{\gamma}_{t} \subset \tilde{G} / \tilde{K}$.

Let $\delta$ be an $h$-geodesic in $\tilde{H} / \tilde{K}$ with the same endpoints as $\tilde{\gamma}_{t}$ and realizing the distance in $\tilde{H} / \tilde{K}$ between them. By Claim $1, \delta \subset \tilde{G} / \tilde{K}$, and so it is a geodesic in $\tilde{G} / \tilde{K}$. Since $\tilde{G} / \tilde{K}$ has no conjugate points it follows that $\delta=\tilde{\gamma}_{t}$, and the length function $\phi_{t}$ from Definition 2.7 corresponding to the identity map satisfies

$$
\phi_{t}(v)=\operatorname{length}(\delta)=\operatorname{length}\left(\tilde{\gamma}_{t}\right)=\operatorname{length}\left(\gamma_{t}\right)=t .
$$

From this it follows that

$$
\operatorname{stre}\left(\Gamma \backslash H / K, h ; \mathcal{F},\left.h\right|_{\mathcal{F}}\right)=\operatorname{vol}\left(S_{\mathcal{F}} \Gamma \backslash H / K\right)
$$

Now we will prove Claim 1.

By [12] the exponential exp: $\mathfrak{h} \rightarrow \tilde{H}$ maps $\mathfrak{n}$ diffeomorphically onto its image. Moreover, the map

$$
\begin{gathered}
\Phi: \quad \exp (\mathfrak{n}) \times \tilde{L} \rightarrow \tilde{H}, \\
(x, y) \mapsto x y,
\end{gathered}
$$

is a diffeomorphism. Since $\tilde{K} \subset \tilde{L}, \Phi$ induces the diffeomorphism

$$
\begin{gathered}
\Psi: \exp (\mathfrak{n}) \times \tilde{L} / \tilde{K} \rightarrow \tilde{H} / \tilde{K}, \\
(x, y \tilde{K}) \mapsto x y \tilde{K},
\end{gathered}
$$


and under the identification $\Psi$ the natural projection $\exp (\mathfrak{n}) \times \tilde{L} / \tilde{K} \rightarrow \exp (\mathfrak{n})$ induces a submersion

$$
\tilde{\pi}: \tilde{H} / \tilde{K} \rightarrow \exp (\mathfrak{n})
$$

Since the metric on $\tilde{H}$ is right $\tilde{L}$-invariant and since $\Psi$ is given by the product, it easily follows that there is a unique Riemannian metric on $\exp (\mathfrak{n})$ such that $\tilde{\pi}$ is a Riemannian submersion. Moreover, by [12] the canonical projection maps $\exp (\mathfrak{n})$ diffeomorphically onto $\tilde{H} / \tilde{L}=H / L$, and hence via this identification the map $\tilde{\pi}$ is the $\tilde{H}$-invariant submersion that lifts the Riemannian submersion $\pi: H / K \rightarrow H / L$ considered earlier in this proof.

Let $\delta:[0,1] \rightarrow \tilde{H} / \tilde{K}$ be a geodesic such that $\delta(0), \delta(1) \in \tilde{G} / \tilde{K}$ and realizing the length in $\tilde{H} / \tilde{K}$ between its endpoints. By homogeneity we can assume that $\delta(0)=e \tilde{K}$ and $\delta(1)=x \tilde{K}$, where $x \in \exp (\mathfrak{m})$.

Write $\Psi^{-1} \circ \delta=\left(\delta_{1}, \delta_{2}\right)$, so that we have $\delta_{1}(0)=e$ and $\delta_{1}(1)=x$. Now consider the path

$$
\begin{gathered}
\delta_{0}:[0,1] \rightarrow \tilde{H} / \tilde{K}, \\
\delta_{0}(t)=\delta_{1}(t) \tilde{K},
\end{gathered}
$$

which has the same endpoints as $\delta$. By our choice $\Psi^{-1}\left(\delta_{0}\right) \subset \exp (\mathfrak{n}) \times\{e \tilde{K}\}$, and so it follows that $\delta_{0}$ is $\tilde{\pi}$-horizontal. It is also clear that $\tilde{\pi}(\delta)=\tilde{\pi}\left(\delta_{0}\right)$, and hence, $\tilde{\pi}$ being a Riemannian submersion, we have

$$
\operatorname{length}(\delta) \geq \operatorname{length}(\tilde{\pi}(\delta))=\operatorname{length}\left(\tilde{\pi}\left(\delta_{0}\right)\right)=\operatorname{length}\left(\delta_{0}\right) .
$$

Since $\delta_{0}$ has the same endpoints as $\delta$, by the length minimizing property of $\delta$ it follows that $\delta$ is $\tilde{\pi}$-horizontal. Hence $\tilde{\pi}(\delta)$ is a geodesic in $\tilde{H} / \tilde{L}=H / L$, and since $\alpha: G / K \rightarrow H / L$ is totally geodesic, by the commutative diagram

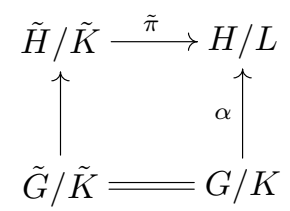

the path $\delta$ lies entirely inside of $\tilde{G} / \tilde{K}$.

To conclude this section we show that diffeomorphisms of foliations have positive stretch, whenever we are dealing with metrics without conjugate points. This will be used for our applications to geometric superrigidity of foliations.

Proposition 2.21. Let $M$ be a compact manifold with a smooth foliation $\mathcal{F}$ carrying a finite invariant transverse measure $\mu$. Assume that $g$ and $h$ are smooth leafwise Riemannian metrics and that $g$ has no conjugate points. If $f$ is a leaf preserving self-diffeomorphism of $M$, then the stretch of $f:(M, \mathcal{F}, g) \rightarrow(M, \mathcal{F}, h)$ is positive.

Proof. Fix $v \in S_{\mathcal{F}} M, t>0$, and let $\gamma_{t}$ be the $g$-geodesic with initial velocity vector $v$ and restricted to $[0, t]$. Let $\alpha$ be an $h$-geodesic homotopic with fixed endpoints to $f\left(\gamma_{t}\right)$, inside the leaf of $M$ containing $f\left(\gamma_{t}\right)$, such that

$$
\phi_{t}(v)=\operatorname{length}_{h}(\alpha)
$$


where $\phi_{t}$ is the function given in Definition 2.7. Since $f$ is a diffeomorphism, there is a path $\alpha_{0}$ in the leaf of $M$ through the basepoint of $v$ homotopic with fixed endpoints to $\gamma_{t}$ such that $f\left(\alpha_{0}\right)=\alpha$, and in particular

$$
\operatorname{length}_{h}(\alpha)=\operatorname{length}_{f^{*} h}\left(\alpha_{0}\right) .
$$

Since $M$ is compact the smooth leafwise Riemannian metrics $g$ and $h$ are quasiisometric, so there is a constant $C>0$ such that

$$
\text { length }_{f^{*} h}\left(\alpha_{0}\right) \geq C \text { length }_{g}\left(\alpha_{0}\right) .
$$

The homotopy between $\gamma_{t}$ and $\alpha_{0}$ lifts to a fixed endpoints homotopy in the universal cover of the leaf containing them, between paths $\tilde{\gamma}_{t}$ and $\tilde{\alpha}_{0}$, where we assume that $\gamma_{t}$ lifts to $\tilde{\gamma}_{t}$ and $\alpha_{0}$ lifts to $\tilde{\alpha}_{0}$. In particular

$$
\operatorname{length}_{g}\left(\alpha_{0}\right)=\operatorname{length}_{g}\left(\tilde{\alpha}_{0}\right) .
$$

Since $\gamma_{t}$ is a $g$-geodesic and $g$ has no conjugate points, it follows that

$$
\operatorname{length}_{g}\left(\tilde{\alpha}_{0}\right) \geq \operatorname{length}_{g}\left(\tilde{\gamma}_{t}\right)=t .
$$

The above relations show that $\phi_{t}(v) \geq C t$, so we conclude that

$$
\operatorname{stre}(f) \geq C \mu_{L}\left(S_{\mathcal{F}} M\right)>0 .
$$

\section{Foliated Heat Flow}

3.1. Preliminaries on vector bundles over foliations. In this subsection we recall some elementary constructions from Riemannian geometry adapted to vector bundles over foliations.

As remarked before, we are assuming $M$ to be a compact manifold with a smooth foliation $\mathcal{F}$ and a finite invariant transverse measure $\mu . M$ also carries a leafwise Riemannian metric $g$, and we denote by $\nabla$ the leafwise Levi-Civita connection defined by $g$. In particular, if $X$ and $Y$ are vector fields on $M$ tangent to the leaves of $\mathcal{F}$, then $\nabla_{X} Y$ coincides with the usual Levi-Civita covariant derivative of $Y$ with respect to $X$ when restricted to each leaf; furthermore we consider such an expression only for vectors tangent to the leaves of $\mathcal{F}$. As usual, $\nabla$ extends to a covariant differential operator on the sections of the tensor algebra of the vector bundle $T_{\mathcal{F}} M$ of leafwise tangent vectors, and we denote such an operator by the same symbol.

Assume that $E \rightarrow M$ is a vector bundle over $M$ which is measurable and leafwise smooth. Let $\nabla^{E}: \mathfrak{X}_{\mathcal{F}}(M) \times \Gamma(E) \rightarrow \Gamma(E)$ be a connection, where $\mathfrak{X}_{\mathcal{F}}(M)$ is the set of measurable leafwise smooth sections of $T_{\mathcal{F}} M$ and $\Gamma(E)$ is the set of measurable leafwise smooth sections of $E$. Let $\langle\cdot, \cdot\rangle_{E}$ be a measurable leafwise smooth $\nabla^{E_{-}}$ parallel metric on $E$.

Denote by $\mathfrak{T}_{\mathcal{F}}^{r, s}(M ; E)$ the set of measurable leafwise smooth sections of the vector bundle $T_{\mathcal{F}} M^{\otimes r} \otimes T_{\mathcal{F}}^{*} M^{\otimes s} \otimes E$. In particular, $\mathfrak{T}_{\mathcal{F}}^{r, s}(M)$ will denote the set of sections of $T_{\mathcal{F}} M^{\otimes r} \otimes T_{\mathcal{F}}^{*} M^{\otimes s}$. Then $\nabla$ and $\nabla^{E}$ induce a covariant differential operator $\nabla$ which maps

$$
\nabla: \mathfrak{T}_{\mathcal{F}}^{r, s}(M ; E) \rightarrow \mathfrak{T}_{\mathcal{F}}^{r, s+1}(M ; E) .
$$


Also, $g$ and $\langle\cdot, \cdot\rangle_{E}$ determine a metric on each vector bundle $T_{\mathcal{F}} M^{\otimes r} \otimes T_{\mathcal{F}}^{*} M^{\otimes s} \otimes E$, which we denote by $\langle\cdot, \cdot\rangle$, and such a metric is $\nabla$-parallel. This in turn induces

$$
\begin{gathered}
\langle\cdot, \cdot\rangle: \mathfrak{T}_{\mathcal{F}}^{r, s}(M ; E) \times \mathfrak{T}_{\mathcal{F}}^{r, s}(M ; E) \rightarrow C_{\mathcal{F}}^{\infty}(M), \\
\langle\alpha, \beta\rangle(x)=\langle\alpha(x), \beta(x)\rangle,
\end{gathered}
$$

where $C_{\mathcal{F}}^{\infty}(M)$ is the set of measurable leafwise smooth functions on $M$. For any of the above we will denote by $|\cdot|$ the norm associated to a metric $\langle\cdot, \cdot\rangle$. Now let $N$ be a compact manifold and $f: M \rightarrow N$ a measurable leafwise smooth map. As before, we assume that either one of the following holds:

(a) $N$ carries a Riemannian metric $h$,

(b) $N$ has a smooth foliation carrying a smooth leafwise Riemannian metric $h$ and $f$ maps leaves into leaves.

We will consider the above remarks for vector bundles given by $E=f^{*}(T N)$ in case (a), and by $E=f^{*}\left(T_{\mathcal{F}} N\right)$ in case (b). In both cases, the pull-back is defined by the leafwise differential of $f$. In particular, the energy density of a map $f$ as above is the measurable leafwise smooth function $e(f): M \rightarrow \mathbb{R}$ given by

$$
e(f)=\frac{1}{2}|d f|^{2},
$$

where $d f$ will always denote the leafwise differential and $|\cdot|$ is the norm constructed above. The total energy of $f$ is then defined by

$$
E(f)=\int_{M} e(f) d \mu d v .
$$

We define a map of vector bundles

$$
\mathrm{P}_{r, s}: T_{\mathcal{F}} M^{\otimes r} \otimes T_{\mathcal{F}}^{*} M^{\otimes s} \otimes E^{\otimes 2} \rightarrow T_{\mathcal{F}} M^{\otimes r} \otimes T_{\mathcal{F}}^{*} M^{\otimes s}
$$

by the assignment

$$
X \otimes \omega \otimes \alpha \otimes \beta \mapsto\langle\alpha, \beta\rangle_{E} X \otimes \omega
$$

and extending it by multilinearity. If $\alpha \in \mathfrak{T}_{\mathcal{F}}^{r, s}(M ; E)$ and $\beta \in \mathfrak{T}_{\mathcal{F}}^{p, q}(M ; E)$, then we define

$$
\alpha \otimes \beta=\mathrm{P}_{r+p, s+q}(\alpha \otimes \beta),
$$

which is an element of $\mathfrak{T}_{\mathcal{F}}^{r+p, s+q}(M)$.

For any connection $\nabla$ on a vector bundle its curvature tensor is defined by

$$
R(X, Y)=\nabla_{X} \nabla_{Y}-\nabla_{Y} \nabla_{X}-\nabla_{[X, Y]} .
$$

If $T \in \mathfrak{T}^{0,4}(P)$, where $P$ is a smooth manifold, then $T$ is said to be of curvature type when the following holds:

$$
\begin{gathered}
T(X, Y, Z, W)=-T(Y, X, Z, W), \\
T(X, Y, Z, W)=T(Z, W, X, Y), \\
T(X, Y, Z, W)+T(X, Z, W, Y)+T(X, W, Y, Z)=0,
\end{gathered}
$$

for any $X, Y, Z$ and $W$. The tensor $T$ is said to be of nonpositive sectional curvature if $T(X, Y, Y, X) \leq 0$ for any $X, Y \in T_{x} P$. Similarly, $T$ is said to be of nonpositive complexified sectional curvature if $T(X, Y, \bar{Y}, \bar{X}) \leq 0$ for any $X, Y \in T_{x}^{\mathbb{C}} P$, where $T$ is extended by complex linearity to $T^{\mathbb{C}} P$. In particular, the sectional curvature and 
complexified sectional curvature of a Riemannian manifold, with curvature tensor $R$, at a plane spanned by $X$ and $Y$ are given by the expressions

$$
\begin{aligned}
K(X, Y) & =\frac{\langle R(X, Y) Y, X\rangle}{|X \wedge Y|^{2}}, \\
K^{\mathbb{C}}(X, Y) & =\frac{\langle R(X, Y) \bar{Y}, \bar{X}\rangle}{|X \wedge Y|^{2}} .
\end{aligned}
$$

3.2. The heat equation and transverse measures. We recollect some of the work of Gromov [11] and Corlette-Zimmer [3] on foliated harmonic theory. They considered the problem of the existence of leafwise harmonic maps through the study of a leafwise heat flow. Even though one has existence of such a heat flow for suitable initial conditions, one may not have convergence since the usual uniform bounds in the case of compact Riemannian manifolds cannot be extended to the case of foliations. In order to bypass some of the difficulties, obtaining only partial results, Gromov [11] introduced a space of functions defined on the leaves of a foliation.

Definition 3.1. Let $M$ be a compact manifold with a smooth foliation $\mathcal{F}$ and a finite invariant transverse measure $\mu$. Assume that $M$ carries a smooth leafwise Riemannian metric $g$ for which the leaves are quotients of a fixed irreducible symmetric space $\Omega$ of noncompact type. Let $N$ be a compact manifold and $f: M \rightarrow N$ a measurable map. We further assume that one of the following is satisfied:

(a) $N$ carries a complete Riemannian metric $h$ and $f$ is leafwise smooth,

(b) $N$ has a smooth foliation $\mathcal{G}$ carrying a smooth leafwise Riemannian metric $h$ which is complete on every leaf, and $f$ is a leaf preserving leafwise smooth map.

Then we define

$$
\mathfrak{M}=\left\{(x, \varphi) \mid x \in M, \varphi: \mathcal{F}_{x} \rightarrow N \text { is smooth }\right\}
$$

where $\mathcal{F}_{x}$ is the leaf in $M$ containing $x$, and we require $\varphi\left(\mathcal{F}_{x}\right)$ to be contained in a leaf of $N$ in case (b) is satisfied. We denote by $\pi: \mathfrak{M} \rightarrow M$ the natural projection $(x, \varphi) \mapsto x$, and define the map

$$
\begin{gathered}
\hat{f}: M \hookrightarrow \mathfrak{M}, \\
x \mapsto\left(x,\left.f\right|_{\mathcal{F}_{x}}\right) .
\end{gathered}
$$

We also denote by $F: \mathfrak{M} \rightarrow N$ the natural evaluation map given by $(x, \varphi) \mapsto \varphi(x)$.

From now on, we will consider fixed the irreducible symmetric space $\Omega ; G$ will denote the identity component of its group of isometries and $K$ will denote a fixed maximal compact subgroup of $G$. The space $\mathfrak{M}$ has a natural equivalence relation given by $(x, \varphi) \sim(y, \psi)$ if and only if $\mathcal{F}_{x}=\mathcal{F}_{y}$ and $\varphi=\psi$. Furthermore, $\mathfrak{M}$ is fairly well behaved, as the following proposition shows.

Proposition 3.2. With $M$ and $N$ as in Definition 3.1, there is a separable metrizable topology on $\mathfrak{M}$ such that the above natural equivalence relation defines a continuous foliation by smooth manifolds for which $\pi$ is a continuous leafwise smooth map. Moreover, $F$ is continuous leafwise smooth, and for any smooth map $f: M \rightarrow N$ as in Definition 3.1 the associated map $\hat{f}: M \hookrightarrow \mathfrak{M}$ is measurable leafwise smooth.

To prove this result we first need the following: 
Lemma 3.3. For $M$ as in Definition 3.1 there is a Borel Map $H: M \times \Omega \rightarrow M$ such that for every $x \in M$,

$$
H\left(\mathcal{F}_{x} \times \Omega\right) \subseteq \mathcal{F}_{x}
$$

and $H_{x}=H(x, \cdot): \Omega \rightarrow \mathcal{F}_{x}$ is a smooth covering satisfying $H(x, o)=x$, where $o=e K$ is the origin in $\Omega=G / K$.

Proof. As remarked in [25], there is a compact manifold $X$, a smooth action on the right of $G$ on $X$ and a principal $K$-bundle $X \rightarrow X / K=M$ so that the pull-back of a leaf in $M$ is a right $G$-orbit in $X$. Moreover, the stabilizer of a point in $X$ is the fundamental group of the leaf containing the projection of such a point onto $M$.

If $g_{1}, g_{2} \in G$ are related by $g_{2}=g_{1} k$ for some $k \in K$, then under the composition of the maps $X \times G \rightarrow X$ and $X \rightarrow M$ given by the action and natural projection, respectively, we have

$$
\begin{aligned}
\left(x, g_{1}\right) & \mapsto x g_{1} \mapsto x g_{1} K, \\
\left(x, g_{2}\right) & \mapsto x g_{2} \mapsto x g_{2} K=x g_{1} k K=x g_{1} K .
\end{aligned}
$$

Since $\Omega=G / K$, the above composition induces a smooth map

$$
\beta: X \times \Omega \rightarrow M .
$$

Now choose a Borel section $\alpha$ of the canonical projection $X \rightarrow X / K=M$. Then the map

$$
\begin{gathered}
H: M \times \Omega \rightarrow M, \\
(x, y) \mapsto \beta(\alpha(x), y),
\end{gathered}
$$

is Borel and clearly maps $H\left(\mathcal{F}_{x} \times \Omega\right) \subseteq \mathcal{F}_{x}$. Moreover, for any $x \in M$ the map $H(x, \cdot): \Omega \rightarrow \mathcal{F}_{x}$ is given by

$$
\begin{gathered}
G / K \rightarrow(\alpha(x) G) / K, \\
g K \mapsto \alpha(x) g K,
\end{gathered}
$$

which is a covering since $\alpha(x) G \cong \Gamma_{x} \backslash G$, where $\Gamma_{x}$ is the stabilizer of $\alpha(x)$ by G.

Proof of Proposition 3.2. Let $H$ be as in Lemma 3.3. Define the map

$$
\begin{gathered}
\Phi: \mathfrak{M} \rightarrow M \times C^{\infty}(\Omega ; N), \\
(m, \phi) \mapsto\left(m, \phi \circ H_{m}\right),
\end{gathered}
$$

which is an injection and induces a topology on $\mathfrak{M}$ by taking the weak topology on $C^{\infty}(\Omega ; N)$, i.e. the topology of uniform convergence on compact sets of partial derivatives. As remarked in [13], the weak topology in $C^{k}(\Omega ; N)$, for $k$ a nonnegative integer, is compatible with a complete separable metric which we may assume bounded. The weak topology in $C^{\infty}(\Omega ; N)$ is the union of the countable collection of topologies given by the inclusions $C^{\infty} \hookrightarrow C^{k}$, and hence it is easily seen to be compatible with a complete separable metric. Therefore, the induced topology on $\mathfrak{M}$ is separable and metrizable. In the case of $N$ a Riemannian manifold we use the globally defined Riemannian metric $h$ to build the (metrizable) weak topology on $C^{\infty}(\Omega ; N)$. In the case of $N$ carrying a leafwise Riemannian metric $h$, we choose a Riemannian metric $h_{1}$ on $N$ which, by compactness of $N$, has leafwise restriction quasi-isometric to $h$; in particular the choice of $h_{1}$ is irrelevant and we can still make computations using $h_{1}$ and its leafwise Levi-Civita connection whenever necessary. 
With this topology $\pi$ is clearly continuous and preserves the leaves, so it induces a continuous foliated structure on $\mathfrak{M}$ by smooth manifolds.

On the other hand, under $\Phi$ the map $F$ corresponds to

$$
\begin{gathered}
\Phi(\mathfrak{M}) \rightarrow N, \\
(m, \alpha) \mapsto \alpha \circ H_{m}^{-1}(m),
\end{gathered}
$$

where $H_{m}^{-1}$ is a local inverse of $H_{m}: \Omega \rightarrow \mathcal{F}_{m}$ in a neighborhood of the origin $o=e K$ of the space $\Omega=G / K$. By Lemma 3.3 we have $\alpha \circ H_{m}^{-1}(m)=\alpha(o)$, so that the map $F$ corresponds to

$$
\begin{gathered}
\Phi(\mathfrak{M}) \rightarrow N, \\
(m, \alpha) \mapsto \alpha(o),
\end{gathered}
$$

which is clearly continuous and leafwise smooth.

Finally, given $f: M \rightarrow N$ smooth, the induced inclusion $\hat{f}: M \rightarrow \mathfrak{M}$ is clearly leafwise smooth. And since $\hat{f}$ corresponds to

$$
\begin{gathered}
M \rightarrow M \times C^{\infty}(\Omega ; N), \\
m \mapsto\left(m, f \circ H_{m}\right),
\end{gathered}
$$

and $H(\cdot, \cdot)=\beta(\alpha(\cdot), \cdot)$, where $\beta$ is smooth and $\alpha$ is measurable, it is easy to check measurability for $\hat{f}$.

Let $f: M \rightarrow N$ be a smooth map as in Definition 3.1. Then we can consider the leafwise heat flow equation

$$
\frac{\partial u}{\partial t}=\Delta u
$$

with initial condition $u_{0}=f$, where $\Delta=-d_{\nabla}^{*} d$ is the leafwise Laplacian, $d_{\nabla}$ the leafwise covariant exterior differential operator on $f^{*}(T N) \otimes T_{\mathcal{F}} M$ coming from the metrics on $M$ and $N$, and $d_{\nabla}^{*}$ is its formal adjoint.

From the work of Li and Tam [15] we have

Proposition 3.4. Let $M, N$ and $f$ be as in Definition 3.1, such that $h$ has nonpositive sectional curvature. If $f: M \rightarrow N$ is a smooth map, then the leafwise heat flow equation with initial condition $f$ has a measurable leafwise smooth solution $f_{t}$ defined on $[0, \infty) \times M$. Moreover, in case $N$ satisfies (b) of Definition 3.1, the maps $f_{t}$ are leaf preserving.

Proof. Since $f$ is smooth, the leafwise energy density $e(f)=\frac{1}{2}|d f|^{2}$ is continuous on $M$ and hence bounded. Then by Theorem 4.1' of [15], the equation has a solution on each leaf, and from the way these are constructed measurability follows.

Remark 3.5. Proposition 3.4 holds true for any compact manifold $M$ as above even if the leafwise Riemannian metric $g$ is not symmetric, as long as $g$ is smooth. The reason for this is that the results in [15] hold for complete Riemannian manifolds with the above curvature condition on $h$ as long as the domain of the maps involved has bounded geometry, which is satisfied in this case since $M$ is compact.

The convergence of the heat flow $f_{t}$ as above cannot be guaranteed. This is mainly because, the leaves being in general noncompact, we cannot ensure the uniform bounds on the derivatives of the heat flow that provide its convergence 
in the case of compact manifolds. However, Gromov [11] showed that certain $L^{2}$ bounds can be obtained. We state the main results on this proved in [11], and refer to [11] and [3] for their proof.

Proposition 3.6. Suppose $u:[0, \infty) \times M \rightarrow N$ is a solution of the heat equation satisfying the hypothesis of Proposition 3.4. Then:

(a) For any $T<\infty$,

$$
\operatorname{ess} \sup _{[0, T] \times M}\left|d u_{t}\right|<\infty \text {. }
$$

(b) The total energy

$$
E\left(u_{t}\right)=\frac{1}{2} \int_{M}\left|d u_{t}\right|^{2} d \mu d v
$$

is a decreasing function of $t$.

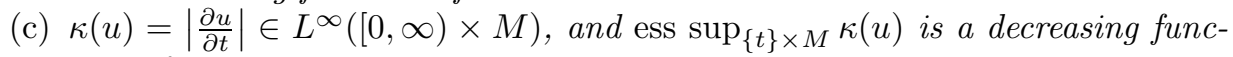
tion of $t$.

Proposition 3.7. Suppose $u:[0, \infty) \times M \rightarrow N$ is a solution of the heat equation satisfying the hypothesis of Proposition 3.4. Then for any non-negative integer $k$ the following hold:

(a) There is a constant $C$ such that

$$
\int_{M}\left|\nabla^{k} d u_{t}\right|^{2} d \mu d v \leq C
$$

(b)

for all $t \geq 0$.

$$
\lim _{t \rightarrow \infty} \int_{M}\left|\nabla^{k} d_{\nabla}^{*} d u_{t}\right|^{2} d \mu d v=0 .
$$

Now consider the maps $\hat{f}_{t}: M \hookrightarrow \mathfrak{M}$ associated to the heat flow $f_{t}$ with initial condition $f$. Since $\hat{f}$ is measurable leafwise smooth, and from the construction of $f_{t}$ in terms of $f$ in Theorems 4.1 and 4.1' of [15], one can check that $\hat{f}_{t}$ is measurable leafwise smooth for every $t$. Then we can consider the one-parameter family of finite invariant transverse measures on $\mathfrak{M}$ given by $\nu_{t}=\left(\hat{f}_{t}\right)_{*}(\mu)$. In the following proposition from Gromov [11] it is proved that this family of measures can be made to subconverge weakly to a measure that singles out harmonic maps from the leaves of $M$ into $N$ or the leaves of $N$ (according to whether $N$ is a Riemannian manifold or a foliation).

Proposition 3.8. Let $M, N$ and a smooth map $f: M \rightarrow N$ satisfy the conditions of Definition 3.1. Assume that $h$ has nonpositive sectional curvature. Then the family of finite invariant transverse measures $\nu_{t}$ is weakly precompact as $t \rightarrow \infty$. Furthermore, any convergent sequence $\left(\nu_{t_{k}}\right)$, with $t_{k} \rightarrow \infty$, has limit $\nu$ supported on the harmonic subfoliation:

$$
\mathfrak{H}=\{(x, \varphi) \in \mathfrak{M} \mid \varphi \text { is harmonic }\} .
$$

Proof. By using the estimate (a) from Proposition 3.7 it is easy to show that there is a constant $C(k, r)$, depending on $k$ and $r$ only, such that for every $t \geq 2$

$$
\int_{m \in M}\left\|\left.\nabla^{k} d f_{t}\right|_{B(m, r)}\right\|_{0}^{2} d \tilde{\mu}(m) \leq C(k, r),
$$


where $B(m, r)$ denotes the ball of radius $r$ centered at $m$ in the leaf $\mathcal{F}_{m}$ passing through $m$. This equation implies that for $t \geq 2$ the set

$$
\left\{m \in M\left|\left\|\left.\nabla^{k} d f_{t}\right|_{B(m, r)}\right\|_{0}^{2}>\frac{2^{k+r+1} C(k, r)}{\delta}\right\}\right.
$$

has $\tilde{\mu}$-measure $<\delta / 2^{k+r+1}$, so that for every $t \geq 2$ the set

$$
K_{\delta}=\left\{m \in M\left|\left\|\left.\nabla^{k} d f_{t}\right|_{B(m, r)}\right\|_{0}^{2} \leq \frac{2^{k+r+1} C(k, r)}{\delta} \forall k \geq 0, \forall r=1,2, \ldots\right\}\right.
$$

satisfies $\tilde{\mu}\left(M \backslash K_{\delta}\right) \leq \delta$. On the other hand, for every $\delta>0$ the set

$$
\mathfrak{K}_{\delta}=\left\{(m, \varphi) \in \mathfrak{M}\left|\left\|\left.\nabla^{k} d \varphi\right|_{B(m, r)}\right\|_{0}^{2} \leq \frac{2^{k+r+1} C(k, r)}{\delta} \forall k \geq 0, \forall r=1,2, \ldots\right\}\right.
$$

is compact, and since $\hat{f}_{t}^{-1}\left(\mathfrak{K}_{\delta}\right)=K_{\delta}$ it follows that $\tilde{\nu}_{t}\left(\mathfrak{M} \backslash \mathfrak{K}_{\delta}\right) \leq \delta$, for every $t \geq 2$. In the notation of [20], the family $\left\{\tilde{\nu}_{t} \mid t \geq 2\right\}$ is uniformly tight and hence precompact in the space of probability measures on $\mathfrak{M}$. Hence any sequence $\left(\tilde{\nu}_{t_{k}}\right)$, with $t_{k} \geq 2$, has a weakly convergent subsequence. Moreover, it is easy to check that for any weak limit $\tilde{\nu}_{t_{k}} \rightarrow \tilde{\nu}$, there is a finite invariant transverse measure $\nu$ such that $\nu_{t_{k}} \rightarrow \nu$ and $\nu$ (together with the leafwise Riemannian measures) integrates to $\tilde{\nu}$. In particular, the family of finite invariant transverse measures $\left\{\nu_{t} \mid t \geq 2\right\}$ is also weakly precompact.

Now let $\nu$ be any weak limit of a sequence $\left(\nu_{t_{k}}\right)$ with $t_{k} \rightarrow \infty$. From the definition of $\nu_{t}$ we get

$$
\int_{\mathfrak{M}}|\Delta \varphi|^{2} d \tilde{\nu}_{t}=\int_{M}\left|\Delta f_{t}\right|^{2} d \tilde{\mu}
$$

and since by Proposition 3.7 the right-hand side of the above identity converges to 0 as $t \rightarrow \infty$, it follows that

$$
\int_{\mathfrak{M}}|\Delta \varphi|^{2} d \tilde{\nu}=0
$$

In other words, $\nu$ is supported on $\mathfrak{H}$.

Although Proposition 3.8 provides the existence of a measure supported on harmonic maps, it might be actually supported on constant maps, making the result uninteresting. By proving heat flow invariance of the stretch we will show that positivity of the stretch for the initial condition map $f$ ensures that some of the maps in the support of $\tilde{\nu}$ are non-constant.

3.3. Heat flow invariance of the stretch. By assuming only measurability we proceed to prove invariance of the stretch under the heat flow.

Proposition 3.9. Let $M, N$ and a smooth map $f: M \rightarrow N$ be as in Definition 2.7. Assume that $M$ has a finite invariant transverse measure $\mu$, that the sectional curvatures of $h$ are nonpositive and that $f$ is a smooth map. If $f_{t}$ is the solution for the heat equation with initial condition $f$, then

$$
\operatorname{stre}(f)=\operatorname{stre}\left(f_{t}\right)
$$

for all $t \geq 0$. 
Proof. By Proposition 3.6 the function $(t, m) \mapsto\left|\frac{\partial f}{\partial t}(t, m)\right|$ is in $L^{\infty}([0, \infty) \times M)$. For fixed $t_{0}>0$, since $M$ has finite volume it follows that

$$
\int_{0}^{t_{0}} \int_{M}\left|\frac{\partial f_{t}}{\partial t}(m)\right| d \mu d v d t<\infty
$$

and hence the function:

$$
H(m)=\int_{0}^{t_{0}}\left|\frac{\partial f_{t}}{\partial t}(m)\right| d t
$$

is integrable on $M$. Let $\phi_{t}$ and $\psi_{t}$ be the functions given in Definition 2.7 and corresponding to $f_{0}=f$ and $f_{t_{0}}$, respectively. Choose $v \in S_{\mathcal{F}} M$ and $t \geq 0$; then when restricted to the leaf of $p(v)$ in $M$ the family of maps $\left\{f_{t}\right\}_{0 \leq t \leq t_{0}}$ defines a smooth homotopy from $f_{0}$ to $f_{t_{0}}$. Let $\gamma_{t}$ be the geodesic in the leaf of $p(v)$ with initial velocity vector $v$ and restricted to $[0, t]$; then the above homotopy of maps when composed with $\gamma_{t}$ defines a homotopy of paths in $N$. Using the definitions of $\phi_{t}$ and $\psi_{t}$ and this homotopy of paths, one can easily show that

$$
\phi_{t}(v) \leq \psi_{t}(v)+\operatorname{length}\left(\alpha_{1}\right)+\operatorname{length}\left(\alpha_{2}\right),
$$

where $\alpha_{1}(s)=f_{s}\left(\gamma_{t}(0)\right)$ and $\alpha_{2}(s)=f_{s}\left(\gamma_{t}(t)\right)$, both defined for $s \in\left[0, t_{0}\right]$. Hence

$$
\operatorname{length}\left(\alpha_{1}\right)=\int_{0}^{t_{0}}\left|\frac{\partial f_{s}}{\partial s}\left(\gamma_{t}(0)\right)\right| d s=H\left(\gamma_{t}(0)\right)=H(p(v)) .
$$

We also have

$$
\operatorname{length}\left(\alpha_{2}\right)=\int_{0}^{t_{0}}\left|\frac{\partial f_{s}}{\partial s}\left(\gamma_{t}(t)\right)\right| d s=H\left(\gamma_{t}(t)\right)=H\left(p\left(g_{t} v\right)\right) .
$$

On the other hand, since $d \mu_{L}$ is $g_{t}$-invariant we have

$$
\int_{S_{\mathcal{F}} M} H\left(p\left(g_{t} v\right)\right) d \mu_{L}(v)=\int_{S_{\mathcal{F}} M} H(p(v)) d \mu_{L}(v)=C_{n-1} \int_{M} H(m) d \mu=C,
$$

where $C$ is a finite positive constant. Hence, after integrating we obtain

$$
\int_{S_{\mathcal{F}} M} \phi_{t}(v) d \mu_{L}(v) \leq \int_{S_{\mathcal{F}} M} \psi_{t}(v) d \mu_{L}(v)+2 C .
$$

Dividing by $t$ and taking the limit as $t \rightarrow \infty$, we obtain $\operatorname{stre}\left(f_{0}\right) \leq \operatorname{stre}\left(f_{t_{0}}\right)$. The reverse inequality is obtained by the same argument, so we have $\operatorname{stre}\left(f_{0}\right)=$ $\operatorname{stre}\left(f_{t_{0}}\right)$.

As a consequence of the above result we obtain

Corollary 3.10. Under the assumptions of Proposition 3.8, if $\operatorname{stre}(f)>0$ then

$$
\nu(\{(m, \varphi) \in \mathfrak{M} \mid \varphi \text { is not constant }\})>0 .
$$

Proof. Let $\left(\nu_{t_{k}}\right)$ be a sequence that converges weakly to $\nu$. By $\left(\mathrm{c}^{\prime}\right)$ of Theorem 4. $D_{1}$ in [11] we have

$$
\int_{(m, \varphi) \in \mathfrak{M}}|d \varphi| d \nu d v=\lim _{t_{k} \rightarrow \infty} \int_{M}\left|d f_{t_{k}}\right| d \mu d v
$$

and by Proposition 2.9 and since $\operatorname{stre}\left(f_{t_{k}}\right)=\operatorname{stre}(f)>0$, for all $t_{k}$, the limit is strictly positive. 


\section{Bochner Formula and Geometric Superrigidity}

4.1. Bochner formula on foliated manifolds. In the previous sections we have established the existence of harmonic maps from the leaves of a foliation into a Riemannian manifold and into the leaves of a manifold foliated by Riemannian manifolds. Moreover, by using the notion of stretch, it is possible to have existence of non-constant harmonic maps. When solving superrigidity problems with geometric techniques for manifolds, after ensuring the existence of suitable harmonic maps, one proceeds to apply a Bochner formula that allows one to turn harmonic maps into totally geodesic maps. This usually requires some kind of restriction on the sign of the curvature of the manifolds involved. A good and rather general example of such application is found in [16]. In this subsection we will show that a Bochner formula as in [16] holds for foliated manifolds, providing a tool for geometric superrigidity on foliated spaces. In what follows we will use the remarks and notations about vector bundles introduced in section 3 .

Remark 4.1. In [16] a tensor $T$ of curvature type is defined to have nonpositive sectional curvature when $T(X, Y, X, Y) \leq 0$, with a similar definition for the complexified sectional curvature. Corresponding to this convention, [16] defines the curvature tensor of a connection $\nabla$ by the expression

$$
R(X, Y)=-\left(\nabla_{X} \nabla_{Y}-\nabla_{Y} \nabla_{X}\right)+\nabla_{[X, Y]} .
$$

Both of these conventions are opposite to those used here, and that accounts for the difference in the way the Bochner formula is expressed here, in Theorem 4.3 below, and in [16].

As a consequence of the main result in [16] we have

Theorem 4.2. Let $M$ be a compact manifold with a smooth foliation $\mathcal{F}$ and a finite invariant transverse measure $\mu$. Let g be a leafwise Riemannian metric which turns each leaf into a quotient of an irreducible symmetric space $\Omega$ of noncompact type, which is either of rank $\geq 2$ or a Cayley or quaternionic hyperbolic space, and denote by $R$ the leafwise curvature tensor. Then there is a smooth $Q \in \mathfrak{T}_{\mathcal{F}}^{0,4}(M)$ satisfying:

1. $Q$ is of curvature type.

2. $\langle Q, R\rangle=0$.

3. $Q$ is leafwise parallel.

4. $\left\langle Q_{x} \circ \sigma_{24}, \alpha \otimes \alpha\right\rangle>0$ for any non-zero symmetric traceless tensor $\alpha$ in $\mathfrak{T}^{0,2}\left(T_{x} \mathcal{F}_{x}\right)$, where $\mathcal{F}_{x}$ is the leaf through $x$ and $\sigma_{24}$ is the permutation defined by $\sigma_{24}(X, Y, Z, W)=(X, W, Z, Y)$.

5. If $\operatorname{rank}(\Omega) \geq 2$, then $\langle Q, T\rangle \geq 0$ for any $T \in \mathfrak{T}_{\mathcal{F}}^{0,4}(M)$ of curvature type with nonpositive sectional curvature.

6. If $\operatorname{rank}(\Omega)=1$, then $\langle Q, T\rangle \geq 0$ for any $T \in \mathfrak{T}_{\mathcal{F}}^{0,4}(M)$ of curvature type with nonpositive complexified sectional curvature.

Proof. A tensor $Q$ as above can be constructed on $\Omega$ as a parallel tensor. Hence it is invariant under the group of isometries of $\Omega$, and so it induces a tensor on every quotient. In particular, each leaf of $M$ carries a tensor with the above properties. Since $g$ is transversally smooth, so is $Q$. 
As before, $N$ will denote a compact smooth manifold and $f: M \rightarrow N$ a measurable leafwise smooth map. Furthermore, we will assume that one of the following is satisfied:

(a) $N$ carries a Riemannian metric $h$,

(b) $N$ has a smooth foliation carrying a smooth leafwise Riemannian metric $h$ and $f$ maps leaves into leaves.

Theorem 4.3. Let $M$ be a compact manifold with a smooth foliation $\mathcal{F}$ and a finite invariant transverse measure $\mu$. Assume that $M$ carries a smooth leafwise Riemannian metric $g$ which turns each leaf into a quotient of $\Omega$ as in Theorem 4.2 with $Q$ the corresponding leafwise parallel tensor. Let $N$ be a compact manifold satisfying the above assumptions. Denote by $R^{N}$ the curvature tensor of $h$, and assume that $R^{N}$ has nonpositive sectional curvature if $\operatorname{rank}(\Omega) \geq 2$ and nonpositive complexified sectional curvature otherwise. If $f: M \rightarrow N$ is a measurable leafwise smooth map (leaf preserving in the case of $N$ foliated) such that its leafwise differential df has square-integrable leafwise covariant derivatives, then

$$
\int_{M}\left\langle Q \circ \sigma_{24}, \nabla d f \otimes \nabla d f\right\rangle d \mu d v=-\frac{1}{2} \int_{M}\left\langle Q, f^{*} R^{N}\right\rangle d \mu d v,
$$

where $\sigma_{24}(x, y, z, w)=(x, w, z, y)$.

Proof. The proof follows the same arguments as the corresponding result in [16] by making use of Stokes' theorem for foliations

With our standing assumptions, if $f: M \rightarrow N$ is a smooth map, then we can consider the leafwise heat flow $f_{t}$. For such a one-parameter family of maps, convergence as $t \rightarrow \infty$ cannot be guaranteed, and a leafwise harmonic map may not be available for applications. However, from (b) of Proposition 3.7 we observe that in a suitable $L^{2}$ space the heat flow is asymptotically harmonic. We will see that the tensor $Q$ of curvature type introduced above allows us to further prove that $f_{t}$ is asymptotically totally geodesic.

Proposition 4.4. Let $M, N$ and $f$ be as in Theorem 4.3. Assume that $f: M \rightarrow N$ is a smooth map and let $f_{t}$ be the associated heat flow. Then

$$
\lim _{t \rightarrow \infty} \int_{M}\left|\nabla d f_{t}\right|^{2} d \mu d v=0 .
$$

Proof. Notice that $\nabla d f_{t} \in \mathfrak{T}_{\mathcal{F}}^{0,2}\left(M ; f_{t}^{*} T N\right)$, and write

$$
\begin{aligned}
& A_{t}=\frac{1}{n} \operatorname{tr}_{g}\left(\nabla d f_{t}\right) g, \\
& B_{t}=\nabla d f_{t}-A_{t},
\end{aligned}
$$

where $n=\operatorname{dim}(\Omega)$. Then $B_{t}$ is traceless and $A_{t}$ is a multiple of $\left(d_{\nabla}^{*} d f_{t}\right) g$. In particular, since $g$ is smooth there is a constant $C$ such that

$$
\left|A_{t}\right| \leq C\left|d_{\nabla}^{*} d f_{t}\right|
$$

and by Proposition 3.7 we have

$$
\lim _{t \rightarrow \infty} \int_{M}\left|A_{t}\right|^{2} d \mu d v=0
$$


It is easy to check that $\left\langle A_{t}, B_{t}\right\rangle=0$, so that $\left|B_{t}\right| \leq\left|\nabla d f_{t}\right|$. On the other hand, since $\tilde{Q}=Q \circ \sigma_{24}$ is smooth on $M$, there is a constant $C_{1}>0$ such that for any $\alpha, \beta \in \mathfrak{T}_{\mathcal{F}}^{0,2}\left(M ; f_{t}^{*} T N\right)$ we have

$$
|\langle\tilde{Q}, \alpha \otimes \beta\rangle| \leq C_{1}|\alpha||\beta| .
$$

In particular, we have the estimates

$$
\begin{aligned}
\left|\int_{M}\left\langle\tilde{Q}, A_{t} \otimes A_{t}\right\rangle d \mu d v\right| & \leq C_{1} \int_{M}\left|A_{t}\right|^{2} d \mu d v \\
\left|\int_{M}\left\langle\tilde{Q}, A_{t} \otimes B_{t}\right\rangle d \mu d v\right| & \leq C_{1}\left(\int_{M}\left|A_{t}\right|^{2} d \mu d v\right)^{1 / 2}\left(\int_{M}\left|B_{t}\right|^{2} d \mu d v\right)^{1 / 2},
\end{aligned}
$$

with a similar relation obtained by interchanging the roles of $A_{t}$ and $B_{t}$ in the last inequality. By Proposition 3.7 we have

$$
\sup _{t} \int_{M}\left|B_{t}\right|^{2} d \mu d v \leq \sup _{t} \int_{M}\left|\nabla d f_{t}\right|^{2} d \mu d v<\infty
$$

Hence the equations (3) and (4) prove that

$$
\begin{aligned}
& \lim _{t \rightarrow \infty} \int_{M}\left\langle\tilde{Q}, A_{t} \otimes A_{t}\right\rangle d \mu d v=0, \\
& \lim _{t \rightarrow \infty} \int_{M}\left\langle\tilde{Q}, A_{t} \otimes B_{t}\right\rangle d \mu d v=0, \\
& \lim _{t \rightarrow \infty} \int_{M}\left\langle\tilde{Q}, B_{t} \otimes A_{t}\right\rangle d \mu d v=0 .
\end{aligned}
$$

By the properties of $Q$ and since $B_{t}$ is traceless, we have $\left\langle\tilde{Q}, B_{t} \otimes B_{t}\right\rangle \geq 0$. Also, by Theorem 4.3

$$
\int_{M}\left\langle\tilde{Q}, \nabla d f_{t} \otimes \nabla d f_{t}\right\rangle d \mu d v=-\frac{1}{2} \int_{M}\left\langle Q, f_{t}^{*} R^{N}\right\rangle d \mu d v \leq 0,
$$

where the last inequality follows from the curvature hypothesis on $N$ and the properties of $Q$. Hence, using the multilinearity of $\otimes$, we have

$$
\begin{aligned}
0 & \leq \int_{M}\left\langle\tilde{Q}, B_{t} \otimes B_{t}\right\rangle d \mu d v \\
& \leq-\int_{M}\left\langle\tilde{Q}, A_{t} \otimes A_{t}\right\rangle d \mu d v-\int_{M}\left\langle\tilde{Q}, A_{t} \otimes B_{t}\right\rangle d \mu d v-\int_{M}\left\langle\tilde{Q}, B_{t} \otimes A_{t}\right\rangle d \mu d v,
\end{aligned}
$$

which by the equations in (5) implies that

$$
\lim _{t \rightarrow \infty} \int_{M}\left\langle\tilde{Q}, B_{t} \otimes B_{t}\right\rangle d \mu d v=0 .
$$

On the other hand, by property 4 of Theorem 4.2 for every $x \in M$ there is a $C_{2}>0$ such that $|\alpha|^{2} \leq C_{2}\left\langle\tilde{Q}_{x}, \alpha \bigotimes \alpha\right\rangle$, for any symmetric traceless (0,2)-tensor at $x$. Since $\tilde{Q}$ is leafwise parallel we can choose $C_{2}$ constant along every leaf of $M$. And since all leaves homothetic with $g$ are smooth and $M$ is compact, we can choose a constant $C_{2}$ which provides the above estimate at every point of $M$. Hence

$$
\int_{M}\left|B_{t}\right|^{2} d \mu d v \leq C_{2} \int_{M}\left\langle\tilde{Q}, B_{t} \otimes B_{t}\right\rangle d \mu d v \rightarrow 0 .
$$

This limit together with $\nabla d f_{t}=A_{t}+B_{t}$ and equation (3) proves the proposition.

As an immediate corollary to this result we have 
Proposition 4.5. Let $M, N$ and $f$ be as in Theorem 4.3. Assume that $f: M \rightarrow N$ is a smooth map and let $f_{t}$ be the associated heat flow. Then there is a sequence of positive real numbers $\left(t_{k}\right)$ such that:

$$
\lim _{k \rightarrow \infty}\left|\nabla d f_{t_{k}}(x)\right|=0
$$

for $\tilde{\mu}$-a.e. point $x \in M$, where $\tilde{\mu}$ is the measure on $M$ obtained by integrating $\mu$ with respect to the leafwise Riemannian measures.

Proof. By Proposition 4.4 we can choose a sequence of positive real numbers $\left(t_{k}\right)$ such that

$$
\int_{M}\left|\nabla d f_{t_{k}}\right|^{2} d \mu d v \leq \frac{1}{2^{k}} .
$$

For such a choice the conclusion follows easily.

4.2. Applications to the geometry of foliations and Riemannian manifolds. As a consequence of the results proved before we have the following refinement of Proposition 3.8:

Theorem 4.6. Let $M$ be a compact manifold with a smooth foliation $\mathcal{F}$ carrying a finite invariant transverse measure $\mu$. Assume that $M$ has a smooth leafwise Riemannian metric $g$ which turns each leaf into a quotient of an irreducible symmetric space $\Omega$ of noncompact type, which is either of rank $\geq 2$ or a quaternionic or Cayley hyperbolic space. Let $N$ be a compact manifold and $f: M \rightarrow N$ a smooth map, and assume that one of the following is satisfied:

(a) $N$ carries a Riemannian metric $h$, or

(b) $N$ has a smooth foliation carrying a smooth leafwise Riemannian metric $h$ and $f$ is leaf preserving.

Assume that $h$ has nonpositive sectional curvature for $\operatorname{rank}(\Omega) \geq 2$ and nonpositive complexified sectional curvature otherwise. Then the family of finite invariant transverse measures $\nu_{t}$ on $\mathfrak{M}$ (as introduced in Proposition 3.8) has a subsequence $\left(\nu_{t_{k}}\right)$, with $t_{k} \rightarrow+\infty$, that weakly converges to a finite invariant transverse measure $\nu$ supported on $\mathfrak{G}=\{(x, \phi) \in \mathfrak{M} \mid \phi$ is totally geodesic $\}$, the totally geodesic subfoliation.

Proof. In what follows, for every $\delta>0$ the set $\mathfrak{K}_{\delta}$ will denote the compact subset of $\mathfrak{M}$ as defined in the proof of Proposition 3.8.

By Proposition 4.5 there is a sequence of positive real numbers $t_{k} \rightarrow \infty$ such that for $\tilde{\mu}$-a.e. $x \in M$

$$
\lim _{k \rightarrow \infty}\left|\nabla d f_{t_{k}}(x)\right|=0 .
$$

Then Egorov's theorem implies that for every $\delta>0$ there is a compact subset $C_{\delta}$ of $M$, with $\tilde{\mu}\left(M \backslash C_{\delta}\right)<\delta$ and such that

$$
\lim _{k \rightarrow \infty}\left|\nabla d f_{t_{k}}\right|=0
$$

uniformly on $C_{\delta}$.

Since the natural projection $\pi: \mathfrak{M} \rightarrow M$ is continuous and $\pi_{*}\left(\nu_{t}\right)=\mu$ for every $t$, we have from the proof of Proposition 3.8 that, for the closed set $\mathfrak{C}_{\delta}=\pi^{-1}\left(C_{\delta}\right)$,

$$
\tilde{\nu}_{t}\left(\mathfrak{M} \backslash \mathfrak{C}_{\delta} \cap \mathfrak{K}_{\delta}\right) \leq \tilde{\nu}_{t}\left(\mathfrak{M} \backslash \mathfrak{C}_{\delta}\right)+\tilde{\nu}_{t}\left(\mathfrak{M} \backslash \mathfrak{K}_{\delta}\right)<2 \delta
$$


for every $t \geq 2$ and for every $\delta>0$, where $\tilde{\nu}_{t}$ denotes the measure on $\mathfrak{M}$ coming from $\nu_{t}$ and the leafwise volumes. But when restricted to $\mathfrak{C}_{\delta} \cap \mathfrak{K}_{\delta}$ the measures $\tilde{\nu}_{t}$ have support contained in

$$
\mathfrak{N}_{\delta}=\overline{\bigcup_{k=0}^{\infty}\left(\mathfrak{C}_{\delta} \cap \mathfrak{K}_{\delta} \cap \hat{f}_{t_{k}}(M)\right)} .
$$

Hence, we have $\tilde{\nu}_{t}\left(\mathfrak{M} \backslash \mathfrak{N}_{\delta}\right)<2 \delta$ for every $\delta>0$. Then the family of measures $\left\{\tilde{\nu}_{t} \mid t \geq 2\right\}$ is uniformly tight with respect to the compact subsets $\mathfrak{N}_{\delta} \subset \mathfrak{M}$. In particular, by passing to a subsequence, we can assume that the sequence $\left(\tilde{\nu}_{t_{k}}\right)$ weakly converges to a finite measure $\tilde{\nu}$, where $\left(t_{k}\right)$ is the sequence for which equation (7) holds. Observe that since uniform convergence is inherited by subsequences, the property of the sets $C_{\delta}$ expressed in equation (8) remains valid.

As in the proof of Proposition 3.8, let $\nu$ be the finite invariant transverse measure on $\mathfrak{M}$ which, together with the leafwise Riemannian measures, integrates to $\tilde{\nu}$. Then, we have the weak limit $\nu_{t_{k}} \rightarrow \nu$.

The set $\mathfrak{N}_{\delta}$ can be rewritten as

(10) $\mathfrak{N}_{\delta}=\left\{(x, \phi) \in \mathfrak{M}\left|\exists\left(x_{j}, f_{t_{k(j)}} \mid \mathcal{F}_{x_{j}}\right) \in \mathfrak{C}_{\delta} \cap \mathfrak{K}_{\delta} \ni x_{j} \rightarrow x, f_{t_{k(j)}}\right| \mathcal{F}_{x_{j}} \stackrel{C^{\infty}}{\longrightarrow} \phi\right\}$,

where $\stackrel{C^{\infty}}{\longrightarrow}$ denotes convergence in the topology of $\mathfrak{M}$, and $\left(t_{k(j)}\right)$ is a sequence taken from the set of real numbers $\left\{t_{k} \mid k=1,2, \ldots\right\}$. Since the limit $\nu_{t_{k}} \rightarrow \nu$ is not affected by dropping finitely many terms, we can assume that $\tilde{\nu}$ restricted to $\mathfrak{N}_{\delta}$ is supported on $\mathfrak{N}_{\delta}^{o}$ defined by $(10)$, where we now assume $\left(t_{k(j)}\right)$ is a subsequence of $\left(t_{k}\right)$.

For any element $\left(x_{j}, f_{t_{k(j)}} \mid \mathcal{F}_{x_{j}}\right) \in \mathfrak{C}_{\delta}$, by the property expressed in equation (8) we have $\lim _{t_{k(j)} \rightarrow \infty}\left|\nabla d f_{t_{k(j)}}\left(x_{j}\right)\right|=0$, so that for $\tilde{\nu}$-a.e. $(x, \phi) \in \mathfrak{N}_{\delta}^{o}$ we have $\nabla d \phi(x)=0$.

Given a sequence $\delta_{i} \searrow 0$, one can easily check (see [20]) that $\tilde{\nu}$ is supported on

$$
\mathfrak{N}=\bigcup_{i=1}^{\infty} \mathfrak{N}_{\delta_{i}}^{o}
$$

In particular, for $\tilde{\nu}$-a.e. $(x, \phi) \in \mathfrak{M}$ we have $\nabla d \phi(x)=0$. Then since $\nu$ integrates to $\tilde{\nu}$, it follows that for $\nu$-a.e. leaf in $\mathfrak{M}$ passing through a point $(x, \phi)$ the map $\phi$ is totally geodesic on a conull set of the leaf. As any such $\phi$ is smooth, we conclude that for $\nu$-a.e. leaf passing through a point $(x, \phi)$ the map $\phi$ is totally geodesic. In other words, the finite invariant transverse measure $\nu$ is supported on the totally geodesic subfoliation $\mathfrak{G}$.

Using our previous results on stretch, we obtain the following corollaries.

Corollary 4.7. Under the assumptions of Theorem 4.6, if $\operatorname{stre}(f)>0$ then

$$
\nu(\{(m, \varphi) \in \mathfrak{M} \mid \varphi \text { is not constant }\})>0 .
$$

Proof. This is an immediate consequence of Corollary 3.10.

Theorem 4.8. Let $M, N$ and $f$ be as in Theorem 4.6. If stre $(f)>0$, then there is an isometric totally geodesic immersion of $\Omega$ into $N$, for $N$ a Riemannian manifold, or into a leaf of $N$, for $N$ a foliated manifold. 
Proof. By Corollary 4.7 and Theorem 4.6 there is a non-constant totally geodesic map from a leaf of $M$ into $N$ or a leaf of $N$, according to whether $N$ is a Riemannian manifold or a foliation. Since the leaves of $M$ are isometrically covered by $\Omega$, and by its irreducibility, it follows that such a map is an isometric (up to a constant) totally geodesic immersion.

Proposition 4.9. Let $M$ be a compact manifold with a smooth foliation carrying a smooth leafwise Riemannian metric $g$ and a finite invariant transverse measure $\mu$. Let $(N, h)$ be a compact Riemannian manifold and $f: M \rightarrow N$ a smooth map. Assume that each leaf of $M$ is isometrically covered by an irreducible symmetric space $\Omega$ of noncompact type, which is either of rank $\geq 2$ or a Cayley or quaternionic hyperbolic space. Assume also that $N$ has nonpositive sectional curvature in case $\operatorname{rank}(\Omega) \geq 2$ and nonpositive complexified sectional curvature otherwise. If $M$ has positive stretch, $f(M)$ is a submanifold and $f$ has a lift $\tilde{f}: \tilde{M} \rightarrow \tilde{N}$ to universal covers which is an embedding onto a bounded distortion submanifold of $\tilde{N}$, then there is an isometric totally geodesic embedding $\Omega \hookrightarrow \tilde{N}$.

Proof. By Proposition 2.14 the stretch of $f$ is non-zero, so the result follows from Theorem 4.8 .

Remark 4.10. We observe that by Theorem 2.20 , if $G$ is the identity component of the group of isometries of $\Omega$ as above, $G \hookrightarrow H$ is a closed inclusion for $H$ semisimple centerless Lie group without compact factors and $\Gamma \subset H$ a cocompact lattice, then the foliated manifold $\Gamma \backslash H / K$ is positively stretched for any maximal compact subgroup $K \subset G$. In particular, Proposition 4.9 can be applied with $M=\Gamma \backslash H / K$ for suitable maps $f$.

The following elementary remark provides an application of the previous arguments to Riemannian manifolds.

Remark 4.11. Let $\tilde{M}$ be a complete simply connected Riemannian manifold and $\tilde{M}=M_{0} \times M_{1} \times \cdots \times M_{k}$ be its de Rham decomposition, where $M_{0}$ is Euclidean and each $M_{i}$ is an irreducible Riemannian manifold for $i=1, \ldots, k$. If $\gamma \in \operatorname{Isom}(\tilde{M})$, then by Theorem 5.4 on page 185 of [14] it follows that $\gamma$ permutes the involutive distributions associated to the irreducible factors of the de Rham decomposition of $\tilde{M}$. In particular $\gamma$ permutes the factors of the de Rham decomposition.

Let $\Gamma$ be a subgroup of $\operatorname{Isom}(\tilde{M})$, and consider the homomorphism $\rho: \Gamma \rightarrow S_{k}$ that assigns to each isometry the corresponding permutation of the irreducible de Rham factors of $\tilde{M}$, where $S_{k}$ denotes the group of permutations of a set with $k$ elements. Then the kernel $\Gamma_{0}=\operatorname{ker}(\rho)$ is a normal finite index subgroup of $\Gamma$ that fixes the de Rham decomposition of $\tilde{M}$, including the order of the factors. Hence the foliations of $\tilde{M}$ induced by the irreducible factors are $\Gamma_{0}$-equivariant.

In particular, if $M$ is a compact (finite volume) quotient of $\tilde{M}$; then $M$ has a finite index Galois covering $M^{\prime}$, and hence compact (of finite volume, resp.), such that the foliations of $\tilde{M}$ defined by the irreducible factors of its de Rham decomposition induce foliations on $M^{\prime}$. Moreover, any such foliation carries a finite invariant transverse measure coming from the Riemannian measures on the complementary factors.

Theorem 4.12. Let $(M, g)$ be a compact connected Riemannian manifold such that its universal cover $(\tilde{M}, g)$ has as a de Rham factor an irreducible symmetric space 
$\Omega$ of noncompact type which is either of rank $\geq 2$ or a Cayley or quaternionic hyperbolic space. If $h$ is a Riemannian metric on $M$ with nonpositive sectional curvature in the case of $\operatorname{rank}(\Omega) \geq 2$ and with nonpositive complexified sectional curvature otherwise, then the Riemannian universal cover of $(M, h)$ has an irreducible de Rham factor containing $\Omega$ by an isometric totally geodesic embedding. In particular, if $\operatorname{rank}(\Omega) \geq 2$ and all rank 1 factors of $(\tilde{M}, h)$ have strictly negative sectional curvature, then through every point of $(M, h)$ there is an isometric and totally geodesic immersed copy of $\Omega$.

Proof. By Remark 4.11 we can replace $M$ by a finite cover to assume that it satisfies the hypothesis of Proposition 4.9, then by Proposition 2.18 it is positively stretched. For $f=i d$ the rest of the conditions in Proposition 4.9 are clear, so there is an isometric totally geodesic embedding $\Omega \hookrightarrow(\tilde{M}, h)$.

If $X$ is a de Rham factor of $(\tilde{M}, h)$, then by taking composition with the natural projection we have a totally geodesic map $\Omega \rightarrow X$. At least one such map is nonconstant, and when this holds we have (by irreducibility of $\Omega$ ) an isometric totally geodesic embedding $\Omega \hookrightarrow X$. In particular we can assume that such a map exists for an irreducible de Rham factor $X_{0}$.

If every rank 1 factor of $(\tilde{M}, h)$ has strictly negative sectional curvature, then $X_{0}$ must have rank $\geq 2$. Since $(M, h)$ is compact with nonpositive sectional curvature, by [7] the Riemannian manifold $X_{0}$ is symmetric. By homogeneity, the inclusion of symmetric spaces $\Omega \hookrightarrow X_{0}$ can be used to show that through every point of $X_{0}$ there is an isometric and totally geodesic embedded copy of $\Omega$. This remark clearly implies the last claim of the statement.

In order to improve the consequences of Theorem 4.8 for leaf preserving maps between foliations we will use the following lemma.

Lemma 4.13. Let $M, N$ and $f$ be as in Theorem 4.6 satisfying case (b). Assume $N$ carries a finite invariant transverse measure $\rho$ and that $f$ preserves the transverse measures, i.e. $f_{*}(\mu)=\rho$. Then for $F: \mathfrak{M} \rightarrow N$ the map given in Definition 3.1 and $\nu$ the finite invariant transverse measure of Theorem 4.6 we have $F_{*}(\nu)=\rho$.

Proof. Let $f_{t}$ be the heat flow associated to $f$; then by the definition of $F$ the following diagram is commutative:



Since the heat flow is leaf preserving, every $f_{t}$ defines the same map between transversals given by $f$. In particular, we have $\left(f_{t}\right)_{*}(\mu)=\rho$, so that by the above diagram we conclude that

$$
F_{*}\left(\nu_{t}\right)=F_{*}\left(\left(\hat{f}_{t}\right)_{*}(\mu)\right)=\left(F \circ \hat{f}_{t}\right)_{*}(\mu)=\left(f_{t}\right)_{*}(\mu)=\rho .
$$

In Theorem 4.6, the transverse measure $\nu$ is a weak limit of a sequence $\left(\nu_{t_{k}}\right)$, and since we have $F_{*}\left(\nu_{t_{k}}\right)=\rho$ for each $t_{k}$, it follows that $F_{*}(\nu)=\rho$.

Theorem 4.14. Let $M$ and $N$ be compact manifolds with smooth foliations carrying smooth leafwise Riemannian metrics $g$ and $h$, respectively, and finite invariant 
transverse measures $\mu$ and $\rho$, respectively. Assume that each leaf of $M$ is isometrically covered by an irreducible symmetric space $\Omega$ of noncompact type which is either of rank $\geq 2$ or a Cayley or quaternionic hyperbolic space. Also assume that $h$ has nonpositive sectional curvature in case $\operatorname{rank}(\Omega) \geq 2$ and nonpositive complexified sectional curvature otherwise. If $f: M \rightarrow N$ is a smooth leaf preserving map with stre $(f)>0$ and that preserves the transverse measures, i.e. $f_{*}(\mu)=\rho$, then there is an isometric (up to a constant) totally geodesic immersion of $\Omega$ into a.e. leaf of $N$.

Proof. Let $\mu$ and $\rho$ be the finite invariant transverse measures of $M$ and $N$, respectively. Let $\nu$ be the finite invariant transverse measure of $\mathfrak{M}$ from Theorem 4.6, so that by Lemma 4.13 we have $F_{*}(\nu)=\rho$.

Since stre $(f)>0$, by Corollary 3.10 there is a leaf-satured subset $\mathfrak{M}_{0}$ of $\mathfrak{M}$, with positive $\nu$-measure, such that for every $(x, \phi) \in \mathfrak{M}_{0}$ the map $\phi$ is a non-constant totally geodesic map from a leaf of $M$ into a leaf of $N$. Hence, from the condition $F_{*}(\nu)=\rho$ we conclude that the leaf saturation $L$ of the subset $F\left(\mathfrak{M}_{0}\right)$ of $N$ has positive $\rho$-measure and is such that there is a non-constant totally geodesic map from $\Omega$ into every leaf contained in $L$. Moreover, any such map is (by irreducibility of $\Omega$ ) an isometric totally geodesic immersion. For ergodic transverse measures the result follows, since $L$ must be conull in this case. In the general case, we can apply this argument to ergodic decompositions of the transverse invariant measures, whose terms are preserved by $f$ and every map $f_{t}$.

As a corollary of Theorem 4.14 we obtain the following metric rigidity theorem for leafwise Riemannian metrics which are locally symmetric.

Theorem 4.15. Let $M$ be a compact manifold with a smooth foliation $\mathcal{F}$, a finite invariant transverse measure and a smooth leafwise Riemannian metric g. Assume that each leaf is isometrically covered by an irreducible symmetric space $\Omega$ of noncompact type, which is either of rank $\geq 2$ or a Cayley or quaternionic hyperbolic space. Let $h$ be a smooth leafwise Riemannian metric on $M$ which has nonpositive sectional curvature if $\operatorname{rank}(\Omega) \geq 2$, and nonpositive complexified sectional curvature otherwise. Then $g$ and $h$ are homothetic on $\mu$-a.e. leaf of $M$.

Proof. By Proposition 2.21 the stretch of the identity map is positive. Hence the result follows from Theorem 4.14.

\section{Concluding Remarks}

5.1. Positive stretch and topologically engaging actions. The notion of a positively stretched manifold was introduced to deal with the problem of foliated heat flow. More specifically, a positively stretched manifold provides non-trivial harmonic maps defined on some of its leaves, as Corollaries 3.10 and 2.15 show. We used a foliated Bochner formula to improve these results to obtain non-trivial totally geodesic maps defined on the leaves of a foliation. This in turn provided a solution to our superrigidity problems for foliations formulated in Problems A and B. Because of this, it should not be surprising to find some links between positively stretched manifolds and known criteria for a manifold to have rigid properties like the notion of a topologically engaging action considered by Zimmer [26]. In what follows we will show the relation between positive stretch and Zimmer's topological engagement condition. 
As before, let $M$ be a compact manifold with a smooth foliation $\mathcal{F}$, a leafwise Riemannian metric $g$ and a finite invariant transverse measure $\mu$. Assume that a Riemannian metric $h$ on $M$ is given. Then the following lemma holds.

Lemma 5.1. If $\mathrm{p}$-stre $(M, h ; \mathcal{F}, g)(v)>0$ for every $v \in S_{\mathcal{F}} M$, then the g-geodesics in the leaves of the foliation $\tilde{\mathcal{F}}$ in the universal cover $\tilde{M}$ have no accumulation point in $\tilde{M}$ as $t \rightarrow \infty$.

Proof. Assume there is a $v \in S_{\tilde{\mathcal{F}}} \tilde{M}$ such that the $g$-geodesic $\tilde{\gamma}$ determined by $v$ satisfies $\tilde{\gamma}\left(t_{k}\right) \rightarrow p$ for some sequence $\left|t_{k}\right| \rightarrow \infty$. Without loss of generality we can assume that $t_{k} \rightarrow+\infty$. Then after projecting $v$ into $S_{\mathcal{F}} M$ we obtain

$$
\phi_{t_{k}}(v)=d_{(\tilde{M}, h)}\left(\tilde{\gamma}(0), \tilde{\gamma}\left(t_{k}\right)\right) \text {. }
$$

Hence we have

$$
\lim _{k \rightarrow \infty} \frac{\phi_{t_{k}}(v)}{t_{k}}=0
$$

which implies p-stre $(M, h ; \mathcal{F}, g)(v)=0$.

Now let $G$ be a centerless semisimple Lie group without compact factors, and $K \subset G$ a maximal compact subgroup. Assume that there is a locally free right action of $G$ on a compact manifold $X$, with torsionless stabilizers. Then $M=X / K$ is a compact manifold with a smooth foliation $\mathcal{F}$ induced by the $G$-orbits in $X$. Moreover, the natural projection $\pi: X \rightarrow M$ is a principal $K$-bundle and $M$ carries a leafwise Riemannian metric $g$ such that each leaf is isometrically covered by the symmetric space $\Omega=G / K$.

We recall that the action of $G$ on $X$ is called topologically engaging if, for the lifted action $\tilde{X} \times \tilde{G} \rightarrow \tilde{X}$ to universal covers, there is a $\tilde{\lambda} \in \tilde{G}$ whose orbits in $\tilde{X}$ are locally closed and $\tilde{\lambda}$ does not project to a compact subgroup of $G$.

Proposition 5.2. For $G, X, M$ and $g$ as above, if for some Riemannian metric $h$ in $M$ we have

$$
\mathrm{p}-\operatorname{stre}(M, h ; \mathcal{F}, g)(v)>0
$$

for every $v \in S_{\mathcal{F}} M$, then the action of $G$ on $X$ is topologically engaging.

Proof. Let $\mathfrak{g}=\mathfrak{k}+\mathfrak{m}$ be a Cartan decomposition of the Lie algebra $\mathfrak{g}=\operatorname{Lie}(G)$ satisfying $\mathfrak{k}=\operatorname{Lie}(K)$. Choose $v \in \mathfrak{m} \backslash\{0\}$ and let $\tilde{\lambda}=\exp (v) \in \tilde{G}$.

Since $\tilde{\lambda}$ does not project to a compact subgroup of $G$, it suffices to show that the $\tilde{\lambda}$-orbits in $\tilde{X}$ are locally closed. In fact, we will prove that the one-parameter subgroup $\tilde{\lambda}_{t}=\exp (t v)$ has closed orbits in $\tilde{X}$.

Suppose that there is an $\tilde{x}_{0} \in \tilde{X}$ so that the $\tilde{\lambda}_{t}$-orbit through $\tilde{x}_{0}$ is not closed. Then there exists $\left|t_{k}\right| \rightarrow \infty$ such that

$$
\tilde{\alpha}\left(t_{k}\right) \rightarrow p \notin\left\{\tilde{x}_{0} \exp (t v)\right\}_{t},
$$

where $\tilde{\alpha}(t)=\tilde{x}_{0} \exp (t v)$.

Consider the commutative diagram

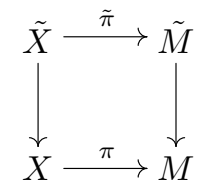

where $\tilde{\pi}$ is a lift to universal covers. 
Let $\alpha$ be the path in $X$ induced by projecting $\tilde{\alpha}$; in other words, $\alpha(t)=x_{0} \exp (t v)$ where $x_{0}$ is the projection of $\tilde{x}_{0}$ under the universal cover map $\tilde{X} \rightarrow X$. Consider the path $\gamma=\pi \circ \alpha$, which is given by $\gamma(t)=x_{0} \exp (t v) K$. It easy to check that, by the choice of the leafwise Riemannian metric $g$ and the basic theory of symmetric spaces, the path $\gamma$ is a $g$-geodesic in a leaf in $M$.

By the above commutative diagram, the path $\tilde{\gamma}=\tilde{\pi} \circ \tilde{\alpha}$ is a lift of $\gamma$ and hence a $g$-geodesic in $\tilde{M}$. Moreover, it satisfies:

$$
\tilde{\gamma}\left(t_{k}\right) \rightarrow \tilde{\pi}(p),
$$

which contradicts Lemma 5.1. Hence the $\tilde{g}_{t}$-orbits are closed.

5.2. Further developments. Zimmer [26] used the topological engagement assumption to study the fundamental group of a manifold acted upon by a semisimple Lie group. Proposition 5.2 shows that an action that generates a pointwise positively stretched foliation is topologically engaging. Hence if we view positive stretch, i.e. $\operatorname{stre}(M, h ; \mathcal{F}, g)>0$, as a weaker but similar condition to that of topological engagement we can proceed to study the fundamental group of a manifold with a positively stretched foliation. In a sense, the main idea is to consider $\operatorname{stre}(M, h ; \mathcal{F}, g)>0$ as implying a sort of topological engagement property on the average. Now we formulate a series of conjectures that come out of these remarks.

Gromov [10] proved that if $G$ is a semisimple Lie group with finite fundamental group and no compact factors acting real analytically on a compact manifold $X$ preserving a unimodular rigid structure of algebraic type (e.g. a pseudo-Riemannian metric), then the action is topologically engaging. Based essentially on this fundamental result, Spatzier and Zimmer proved the following:

Theorem (Spatzier-Zimmer [21]). Let $G$ be connected Lie group with finite center, finite fundamental group and with $\mathbb{R}-$ rank $\geq 2$. Let $X$ be a compact manifold and suppose there is a real analytic action of $G$ on $X$ preserving a real analytic connection and a finite measure. Then $\pi_{1}(X)$ is not isomorphic to the fundamental group of any complete Riemannian manifold $N$ with negative curvature bounded away from 0 and $-\infty$.

Based on this, we expect that in the above theorem one can replace the existence of an invariant connection by the condition of $M=X / K$ being a positively stretched manifold. More precisely:

Conjecture. Let $G$ be a connected centerless noncompact simple Lie group of $\mathbb{R}$ rank $\geq 2$, and $K$ a maximal compact subgroup of $G$. Let $X$ be a compact manifold and suppose there is a locally free action of $G$ on $X$ preserving a finite measure and such that $M=X / K$ is a positively stretched foliated manifold. Then $\pi_{1}(X)$ is not isomorphic to the fundamental group of any complete Riemannian manifold $N$ with negative curvature bounded away from 0 and $-\infty$.

Related to this setup, one can consider the statement obtained by replacing $X$ by a compact foliation, without actually considering an action:

Conjecture. Let $M$ be a compact manifold with a smooth foliation carrying a smooth leafwise Riemannian metric $g$ and a finite invariant transverse measure. Assume that each leaf is isometrically covered by an irreducible symmetric space $\Omega$ of rank $\geq 2$. If $M$ is positively stretched, then $\pi_{1}(M)$ is not isomorphic to the 
fundamental group of any complete Riemannian manifold $N$ with negative curvature bounded away from 0 and $-\infty$.

This conjecture would follow for $N$ compact from Theorem 4.8 as long as one could guarantee the existence of a map $f: M \rightarrow N$ whose stretch is bounded from below by the stretch of $M$ - this is a consequence of the hypothesis $\pi_{1}(M) \cong \pi_{1}(N)$. This remark suggests a way of improving the conditions we have given for a map or a foliation to be positively stretched. Related to this problem, we expect the following to hold:

Conjecture. Let $M$ and $N$ be compact manifolds with smooth foliations carrying smooth leafwise Riemannian metrics and finite invariant transverse measures. Let $f: M \rightarrow N$ be a smooth leaf preserving map. If $f$ defines a measure preserving transverse equivalence, then $\operatorname{stre}(f)>0$.

Such a result would improve Theorems 4.14 and 4.15 to statements more in the spirit of the strong rigidity for foliations from Zimmer [22].

\section{REFERENCES}

1. W. Ballmann and P. Eberlein, Fundamental groups of manifolds of nonpositive curvature, J. Diff. Geom. 25 (1987), 1-22. MR 88b:53047

2. W. Ballmann, M. Gromov, and V. Schroeder, Manifolds of nonpositive curvature, Birkhäuser, Boston, 1985. MR 87h:53050

3. K. Corlette and R. J. Zimmer, Superrigidity for cocycles and hyperbolic geometry, Internat. J. Math. 5 (1994), 273-290. MR 95g:58055

4. C. Croke and A. Fathi, An inequality between energy and intersection, Bull. London Math. Soc. 22 (1990), 489-494. MR 92d:58042

5. P. Eberlein, Isometry groups of simply connected manifolds of nonpositive curvature. II, Acta Math. 149 (1982), 41-69. MR 83m:53055

6. Symmetry diffeomorphism group of a manifold of nonpositive curvature, Trans. Amer. Math. Soc. 309 (1988), 355-374. MR 89i:53028

7. P. Eberlein and J. Heber, A differential geometric characterization of symmetric spaces of higher rank, Inst. Hautes Études Sci. Publ. Math. 71 (1990), 33-44. MR 91j:53022

8. S. Gallot, D. Hulin, and J. Lafontaine, Riemannian geometry, Springer-Verlag, Berlin, 1987. MR 88k:53001

9. C. Godbillon, Feuilletages, Études géométriques, Progress in Mathematics, vol. 98, Birkhäuser, Basel, 1991. MR 93i:57038

10. M. Gromov, Rigid transformations groups, Géométrie Différentielle, Colloque Géométrie et Physique de 1986 en l'honneur de André Lichnerowicz (D. Bernard and Y. Choquet-Bruhat, eds.), Hermann, Paris, 1988, pp. 65-139. MR 90d:58173

11. Foliated Plateau problem. part II: Harmonic maps of foliations, Geom. Funct. Anal. 1 (1991), 253-320. MR 93a:58048

12. S. Helgason, Differential geometry, Lie groups and symmetric spaces, Pure and Applied Mathematics, vol. 80, Academic Press, New York, 1978. MR 80k:53081

13. M. W. Hirsch, Differential topology, Springer-Verlag, New York, 1988. MR 56:6669 (1st ed.)

14. S. Kobayashi and K. Nomizu, Foundations of differential geometry, Vol. I, Wiley, New York, 1963. MR 27:2945

15. P. Li and L. Tam, The heat equation and harmonic maps of complete manifolds, Invent. Math. 105 (1991), 1-46. MR 93e:58039

16. N. Mok, Y.-T. Siu, and S.-K. Yeung, Geometric superrigidity, Invent. Math. 113 (1993), 57-83. MR 94h:53079

17. C. C. Moore and C. Schochet, Global analysis on foliated spaces, Mathematical Sciences Research Institute Publications, vol. 9, Springer-Verlag, New York, 1988. MR 89h:58184

18. G. D. Mostow, Strong rigidity for locally symmetric spaces, Annals of Mathematics Studies, vol. 78, Princeton University Press, Princeton, 1973. MR 52:5874

19. A. L. Onishchik and E. B. Vinberg, Lie groups and algebraic groups, Springer-Verlag, Berlin, 1990. MR 91g:22001 
20. K. R. Parthasarathy, Probability measures on metric spaces, Probability and Mathematical Statistics, vol. 3, Academic Press, New York, 1967. MR 37:2271

21. R. J. Spatzier and R. J. Zimmer, Fundamental groups of negatively curved manifolds and actions of semisimple groups, Topology 30 (1991), 591-601. MR 92m:57047

22. R. J. Zimmer, Strong rigidity for ergodic actions of semisimple Lie groups, Ann. of Math. 112 (1980), 511-529. MR 82j:22011

23. __ Ergodic theory, semisimple Lie groups and foliations by manifolds of negative curvature, Inst. Hautes Études Sci. Publ. Math. 55 (1982), 37-62. MR 84h:22022

24. —_ Ergodic theory and semisimple groups, Birkäuser, Boston, 1984. MR 86j:22014

25. - Arithmeticity of holonomy groups of Lie foliations, J. Amer. Math. Soc. 1 (1988), 35-58. MR 89c:22019

26. __ Representations of fundamental groups of manifolds with a semisimple transformation group, J. Amer. Math. Soc. 2 (1989), 201-213. MR 90i:22021

Department of Mathematics, University of Chicago, 5734 South University Avenue, Chicago, Illinois 60637

Current address: Department of Mathematics, CIEA-IPN, Apartado Postal 14-740, 07300 Mexico DF, Mexico

E-mail address: quiroga@math.cinvestav.mx 